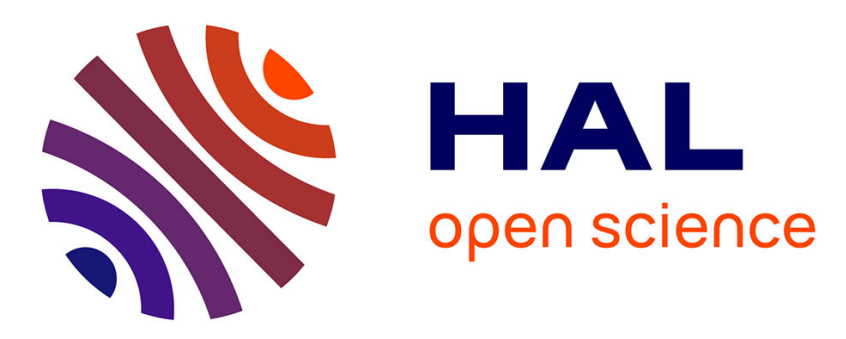

\title{
Gaussian Copula Multivariate Modeling for Image Texture Retrieval Using Wavelet Transforms
}

Nour-Eddine Lasmar, Yannick Berthoumieu

\section{To cite this version:}

Nour-Eddine Lasmar, Yannick Berthoumieu. Gaussian Copula Multivariate Modeling for Image Texture Retrieval Using Wavelet Transforms. IEEE Transactions on Image Processing, 2014, 23 (5), pp.2246 - 2261. 10.1109/TIP.2014.2313232 . hal-00727127v3

\section{HAL Id: hal-00727127 \\ https://hal.inria.fr/hal-00727127v3}

Submitted on 25 Mar 2014

HAL is a multi-disciplinary open access archive for the deposit and dissemination of scientific research documents, whether they are published or not. The documents may come from teaching and research institutions in France or abroad, or from public or private research centers.
L'archive ouverte pluridisciplinaire HAL, est destinée au dépôt et à la diffusion de documents scientifiques de niveau recherche, publiés ou non, émanant des établissements d'enseignement et de recherche français ou étrangers, des laboratoires publics ou privés. 


\title{
Gaussian Copula Multivariate Modeling for
}

\section{Texture Image Retrieval Using Wavelet}

\section{Transforms}

Nour-Eddine Lasmar, and Yannick Berthoumieu, Member, IEEE

\begin{abstract}
In the framework of texture image retrieval, a new family of stochastic multivariate modeling is proposed based on Gaussian Copula and wavelet decompositions. We take advantage of the copula paradigm which makes it possible to separate dependency structure from marginal behavior. We introduce two new multivariate models using respectively generalized Gaussian and Weibull densities. These models capture both the subband marginal distributions and the correlation between wavelet coefficients. We derive, as a similarity measure, a closed form expression of the Jeffrey divergence between Gaussian Copula-based multivariate models. Experimental results on well-known databases show significant improvements in retrieval rates using the proposed method compared to the best known state-of-the-art approaches.
\end{abstract}

Index Terms-Texture, Gaussian Copula, Multivariate generalized Gaussian, Multivariate Weibull, Jeffrey Divergence, Wavelet transforms.

\section{INTRODUCTION}

Characterizing textures is fundamental for various image processing applications ranging from image retrieval to segmentation or compression. In the framework of texture retrieval, the challenge is to provide baseline algorithms making a system able to retrieve, from a textured image databases, the relevant candidates similar to a given query according to the texture cue. A typical

Copyright (c) 2013 IEEE. Personal use of this material is permitted. However, permission to use this material for any other purposes must be obtained from the IEEE by sending a request to pubs-permissions@iee.org.

Manuscript received June 16, 2011; revised June 09, 2012 and November 18, 2013; accepted March 05, 2014. This work was funded by a grant from the Institut Polytechnique de Bordeaux.

N. Lasmar (corresponding author) and Y. Berthoumieu are with the Signal and Image Processing Group,IMS Laboratory, Institut Polytechnique de Bordeaux, France (e-mails: \{nour-eddine.lasmar, yannick.berthoumieu\} @ims-bordeaux.fr). 
retrieval scheme consists of two major tasks. The first one is devoted to feature extraction, where signatures are estimated from each image in the database and from the query. The second task evaluates a similarity measure, based on previous features, to decide which images of the database are close to the query. Thus, one important issue of texture browsing or searching systems is to provide a tractable mathematical description of natural textures. The question of determining textural features with a selective similarity measure has been addressed extensively during the last three decades. The literature on the topic has pointed out that probabilistic approaches are well-founded in terms of retrieval performance [1], [2]. If initially some approaches made use of descriptive statistics such as co-occurrence matrices [3]-[5], recent works have proposed to use explicit parametric random field modeling [1], [2], [6]-[9].

In fact, standard random field modeling consists in providing a parametric probability density functions (PDF) which enables us to fit the empirical histograms of specific visual cues [1], [2], [6]-[9]. The reason is, on the one hand, stochastic model-based approaches are theoretically justifiable since information divergences such as the Kullback-Leibler divergence (KLD) are asymptotic limits of likelihood functions that can be used to measure the similarity between data drawn from different distribution families; the stochastic framework has proven to be asymptotically optimal in terms of the retrieval rate when the KLD between PDF models is used [1], [2]. On the other hand, the parametric models achieve reasonable computational cost for a nearest similar sample search.

In practice, the question of describing a visual content in terms of PDF modeling has been already addressed. Most popular methods show the interest of working with wavelet transforms to project the visual information in a multiscale and multiorientation domain by using filter banks or wavelet transforms [10]-[13]. These approaches are based on studies of human and mammalian vision systems which support that multiscale analysis maximizes simultaneous localization in both spatial and frequency domains [14]-[17]. However, whatever the choice of decomposition, authors pointed out the non-Gaussian behavior of subband coefficients in the wavelet domain [2], [6]-[9]. Many studies propose the generalized Gaussian density (GG) to successfully characterize the marginal distribution of subband coefficients in the wavelet domain [18], [19], [2]. The GG model, jointly used with closed form KLD, leads to significant improvement in retrieval rate over traditional methods based on basic statistics [2]. Srivastava et al. [6] proposed to characterize filter bank outputs by using the Bessel K forms (BKF). In [7], it was conjectured that magnitudes of wavelet coefficients of some classes of textured images have Gamma distribution (Gam). Recent works of Kwitt and Uhl propose to model the detail subband coefficient magnitudes by Weibull distribution (Wbl) [8], [9]. Their approach achieves higher retrieval rates than those using GG or Gam distributions.

All these univariate models lead to a simple and tractable approach. Nevertheless, univariate modeling does not provide a complete statistical description of subband coefficients. While it implies low complexity retrieval systems, it neglects one of the important statistical aspects characterizing a textured image which is the spatial dependency of wavelet coefficients across the 
same subband. For this reason, some authors have started to study the joint statistics of wavelet coefficients via multivariate modeling. Tzagkarakis et al. [20] proposed a computationally complex Gaussianization procedure of filter bank outputs in order to model wavelet coefficients by the multivariate Gaussian distribution. Powerful statistical algorithms have been developed for image denoising using a Multivariate generalized Gaussian distribution (MGGD) [21] or Elliptically Contoured Distribution (ECD) [22], but no closed expression exists for the KLD between these joint distributions to measure similarity in a retrieval or classification context. The responses of filter banks exhibit non-Gaussian joint statistical behavior and the dependency between local coefficients can be captured using Gaussian Scale Mixture (GSM) [23]-[25]. Boubchir et al. [25] recently used the GSM model to define the Multivariate Bessel K Form distribution (MBKF) which is an extension of the univariate model BKF introduced by Srivastava et al. in [6]. The multivariate Gaussian mixture (MGmix) was introduced to model wavelet coefficients or DCT coefficients obtained from overlapping sliding windows [26]-[28]. Since there is no closed form for KLD between two MGmix PDFs, Goldberger et al. used an approximation of KLD to achieve good retrieval rates [27]. For the same reason, in [28], the likelihood was employed for similarity measurement leading to competitive results. More recently, MGmix was used for texture classification and segmentation [29], [30] and other approximations for KLD between MGmix models were proposed [31]. Recently, Verdoolaege and Scheunders introduced the Multivariate Power Exponential (MPE) distribution, also called the Multivariate generalized Gaussian distribution (MGG), as an adequate model for the wavelet statistics of color texture images [32]. Because a closed form of KLD was difficult to find -except the bivariate case- they have considered the Rao geodesic distance on the manifold of MGG distributions as a similarity measure.

Latterly, researchers started to study the multivariate wavelet modeling using copulas [33]-[37]. The wavelet coefficients of multichannel images have been modeled by a copula-based PDF and the normalized Euclidean distance has been employed as similarity measure in a retrieval context [33]. Because of the lack of a closed form expression, the KLD between copula-based models has been approximated by an empirical estimate using a Monte-Carlo (MC) approach [34], [35]. This MC procedure is computationally expensive and thus the KLD closed form, when it exists, is to be preferred [9]. Independently of our work, Kwitt et al. recently took a similar approach where they introduced several copula-based models to characterize the association between subband coefficients from color channels at the same decomposition scale, in the context of color texture retrieval [36]. They claim that no closed form expression of KLD exists in the case of copula-based models; hence they employed likelihood similarity as an alternative strategy to measure similarity between models.

Considering the case of multivariate stochastic modeling in the wavelet domain, the proposed method consists in providing a flexible non-Gaussian model based on Gaussian Copula and the corresponding closed form of KLD for texture classification. As briefly presented in our previous work [37], the main interest of copulas is to allow constructing multivariate distributions to jointly model wavelet coefficients while keeping a good usability for fitting various kinds of marginal distributions. According to 
earlier works on marginal distribution modeling, in this paper, our contribution can be summarized as follows:

- Two multivariate distributions are presented to model spatial dependency of intraband wavelet coefficients; namely, the Gaussian Copula-based Multivariate generalized Gaussian distribution (GC-MGG) and the Gaussian Copula-based Multivariate Weibull distribution (GC-MWbl). Using these two models, we show the ability of the copula approach to easily provide various models suitable for different application domains. Under the assumption that the cumulative distribution function of the marginal is continuous and invertible, any family of marginal distribution can be used to form a multivariate distribution with Gaussian Copula.

- The KLD and its symmetrized version called the Jeffrey divergence (JD) are derived for Gaussian Copula-based multivariate distributions. To our knowledge, this is the first time that the closed form of the KLD is proposed for such multivariate models. This derivation shows that the KLD associated with Gaussian Copula-based models corresponds simply to the contribution of two orthogonal parts. The first part is devoted to evaluating the divergence along the Gaussian manifold due to the Gaussian Copula choice. The second part corresponds to the KLD component resulting from evaluating the divergence along the product of marginal manifold.

The outline of the paper is as follows. In section II, we summarize briefly the statistical retrieval framework and then motivate our choice to model intraband wavelet coefficients using multivariate modeling. In section III, we present the Gaussian Copulabased multivariate modeling and provide an overview of GC-MGG and GC-MWbl models. Hyperparameters estimation is investigated and closed form expressions for the KLD between the models presented are derived in section IV. Experimental results in section $\mathrm{V}$ show the improvement in retrieval rate by using the new approach. Section VI concludes with a discussion and an outlook on future works.

\section{Multivariate Statistical Retrieval}

Vasconcelos and Lippman [1], [26] have defined the statistical retrieval framework and shown that the KLD is the asymptotic limit of the maximum likelihood similarity criteria. In the context of this framework, authors consider wavelet subbands as realizations of scalar random variables and their histograms are defined as univariate probability distribution functions [2], [6][9]. In our approach, multivariate modeling is used to describe wavelet subbands. A natural extension of wavelet subband univariate modeling for texture retrieval is to consider the joint density of a vector of neighboring wavelet coefficients.

\section{A. Multivariate stochastic retrieval framework}

We consider an image database with $M$ images $I_{i}, i=1, \ldots, M$. The goal is to retrieve from a database the top $N$ images $(N \ll M)$ similar to a given query image $I_{q}$. For multivariate modeling purposes, each image $I_{i}$ is represented by a data set $\boldsymbol{X}_{\boldsymbol{i}}=\left\{\vec{x}_{i 1}, \vec{x}_{i 2}, \ldots, \vec{x}_{i L}\right\}$ where $\vec{x}_{i j}, j=1, \ldots, L$ are vectors with elements obtained after a transformation step such as wavelet 
decomposition; $L$ is the number of vector samples considered. Furthermore, each data set $\boldsymbol{X}_{\boldsymbol{i}}$ is assumed to be an independent identically distributed (i.i.d) realization from a multivariate $\operatorname{PDF} p_{i}\left(\vec{x} ; \theta_{i}\right)$ where $\theta_{i}$ contains the hyperparameters of the model. We associate an image index indicator variable $Y \in\{1, \ldots M\}$ to the images in the database.

The optimum rule (in terms of error probability) for retrieving the $N$ relevant images similar to the query image $I_{q}$, selects those which obey

$$
\begin{gathered}
P\left(Y=k_{1} \mid \boldsymbol{X}_{\boldsymbol{q}}\right) \geq P\left(Y=k_{2} \mid \boldsymbol{X}_{\boldsymbol{q}}\right) \geq \cdots \geq P\left(Y=k_{N} \mid \boldsymbol{X}_{\boldsymbol{q}}\right) \\
k_{l} \in\{1, \ldots, M\}, l=1, \ldots, N \text { and } k_{l} \neq k_{m} \text { if } l \neq m
\end{gathered}
$$

From the Bayes' formula $P\left(Y=k_{l} \mid \boldsymbol{X}_{\boldsymbol{q}}\right)=\frac{P\left(\boldsymbol{X}_{\boldsymbol{q}} \mid Y=k_{l}\right) P\left(Y=k_{l}\right)}{P\left(\boldsymbol{X}_{\boldsymbol{q}}\right)}, l=1, \ldots, N$, if the images are $a$ priori equally likely, this optimum rule becomes the ML selection rule [28]

$$
\begin{gathered}
P\left(\boldsymbol{X}_{\boldsymbol{q}} \mid Y=k_{1}\right) \geq P\left(\boldsymbol{X}_{\boldsymbol{q}} \mid Y=k_{2}\right) \geq \cdots \geq P\left(\boldsymbol{X}_{\boldsymbol{q}} \mid Y=k_{N}\right) \\
k_{l} \in\{1, \ldots, M\}, l=1, \ldots, N \text { and } k_{l} \neq k_{m} \text { if } l \neq m
\end{gathered}
$$

where $P\left(\boldsymbol{X}_{\boldsymbol{q}} \mid Y=k_{l}\right)=\prod_{j=1}^{L} p_{k_{l}}\left(\vec{x}_{q j} ; \theta_{k_{l}}\right)$

This likelihood selection rule is equivalent to retrieving images $I_{k_{l}}, l \in\{1, \ldots, N\}$ which maximize $P\left(\boldsymbol{X}_{\boldsymbol{q}} \mid Y=k_{l}\right)$ and then ordering the $N$ relevant images close to the query $I_{q}$.

Vasconcelos and Lippman have shown [1] that in typical asymptotic condition $(L \rightarrow \infty)$, the ML decision rule of (2) is equivalent to

$$
\begin{gathered}
K L D\left(p_{q}\left(\vec{x} ; \theta_{q}\right) \| p_{k_{1}}\left(\vec{x} ; \theta_{k_{1}}\right)\right) \leq K L D\left(p_{q}\left(\vec{x} ; \theta_{q}\right) \| p_{k_{2}}\left(\vec{x} ; \theta_{k_{2}}\right)\right) \leq \cdots \leq K L D\left(p_{q}\left(\vec{x} ; \theta_{q}\right) \| p_{k_{N}}\left(\vec{x} ; \theta_{k_{N}}\right)\right) \\
k_{l} \in\{1, \ldots, M\}, l=1, \ldots, N \text { and } k_{l} \neq k_{m} \text { if } l \neq m
\end{gathered}
$$

Hence, the optimal ML decision rule to select the $N$ top matches to the query image $I_{q}$ is asymptotically equivalent to compute the $K L D\left(p_{q}\left(\vec{x} ; \theta_{q}\right) \| p_{k_{l}}\left(\vec{x} ; \theta_{k_{l}}\right)\right), l=1, \ldots, M$, and then use the decision rule (3) to sort the $N$ top similar images $\left\{I_{k_{1}}, I_{k_{2}}, \ldots, I_{k_{N}}\right\}$

Moreover, taking into account the fact that KLD is asymmetric the symmetrized version JD is more suitable for image retrieval or classification [38]. Thereafter, for evaluating retrieval performance we will use the JD which is defined by

$$
J D\left(p_{q}\left(\vec{x} ; \theta_{q}\right), p_{i}\left(\vec{x} ; \theta_{i}\right)\right)=K L D\left(p_{q}\left(\vec{x} ; \theta_{q}\right) \| p_{i}\left(\vec{x} ; \theta_{i}\right)\right)+K L D\left(p_{i}\left(\vec{x} ; \theta_{i}\right) \| p_{q}\left(\vec{x} ; \theta_{q}\right)\right)
$$

The two steps of retrieval applications (feature extraction and similarity measurement) must also meet the timing constraint and are required to have low computational cost. This requirement remains true for certain parametric approaches which are a computationally efficient implementation [9]. First, finding consistent estimators to ensure convergence of the model's hyperparameter $\hat{\theta}_{i}$ to the true one $\theta_{i}$ is addressed. For instance, the Maximum Likelihood (ML) estimator is defined by 


$$
\hat{\theta}_{i}=\arg \max _{\theta} \sum_{j=1}^{L} \log p\left(\vec{x}_{i j} ; \theta\right)
$$

Second, similarities are computed using the estimated hyperparameters $\hat{\theta}_{\mathrm{q}}$ and $\hat{\theta}_{\mathrm{i}}$. This step must be done in a timely manner, so a closed form expression is required because it is more efficient compared to employing a Monte-Carlo method to estimate KLD and therefore JD. Even if probabilistic approaches can lead to a significant gain in retrieval accuracy, the drawback is its computationally expensive complexity. The optimum selection rule (2) could be high computationally expensive and a reduction of data size $L$ is required to decrease this complexity [26], [36]. However, this comes at the expense of the retrieval accuracy. For these reasons, the closed form of the KLD/JD, when it exists, is more computationally efficient than the use of likelihood similarity.

\section{B. Selecting a multivariate model}

Several authors have studied the dependency between multiscale oriented subband coefficients. Po and Do [39] measured the level of dependency based on mutual information in intraband and interband coefficients. In the framework of image denoising in the wavelet domain, Tan et al. [22] and Portilla et al. [24] proposed various structures for wavelet coefficient neighborhood. All these works point out that the predominant dependency corresponds to the spatial one, i.e. the intraband neighborhood structure. To illustrate the importance of the intraband dependency compared to interorientation and interscale ones, Chi-plot graphs [40] can be used to observe the different types of dependency. Fig. 1 shows a set of Chi-plots for a selection of wavelet coefficient pairs. The Chi-plot can be considered as an extension of the scatterplot. We have used a common setting as it is noted in [40] to define the tolerance band which is shown as a gray-shaded region. A deviation from the tolerance band indicates a dependence structure. We observe that the deviation from tolerance band is more prominent for the intraband dependence than the interorientation or interscale cases. The measures are located inside or closely around the tolerance band for interorientation and interscale wavelet coefficient pairs. Although we cannot categorically claim interorientation and interscale independency, the dependencies across subbands are less important than those within them. We opted for modeling only the intraband dependence even though information can be disregarded for some texture classes.

Note that assuming statistical independency between subbands enables us to use the chain rule [41] for the overall JD derivation between two images. Thus, the whole JD is simply the sum of JDs across subbands. 
Dependence between a wavelet coefficient and its neighbor
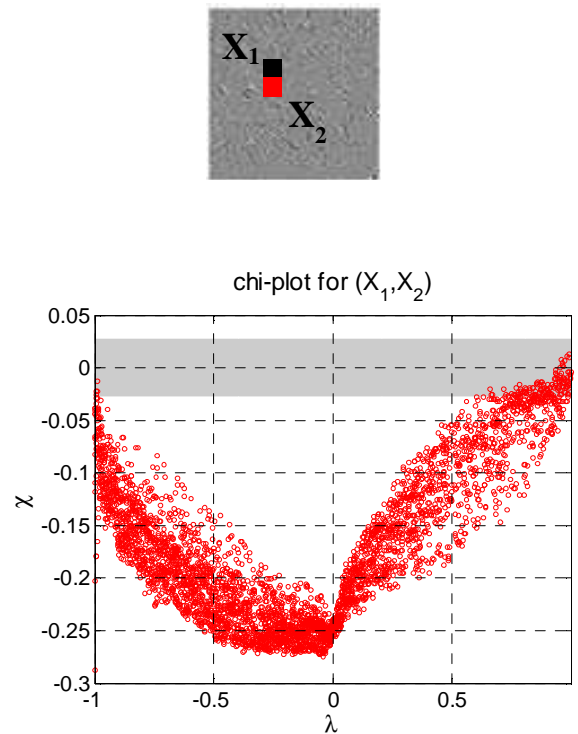

Dependence between a wavelet coefficient and its cousin
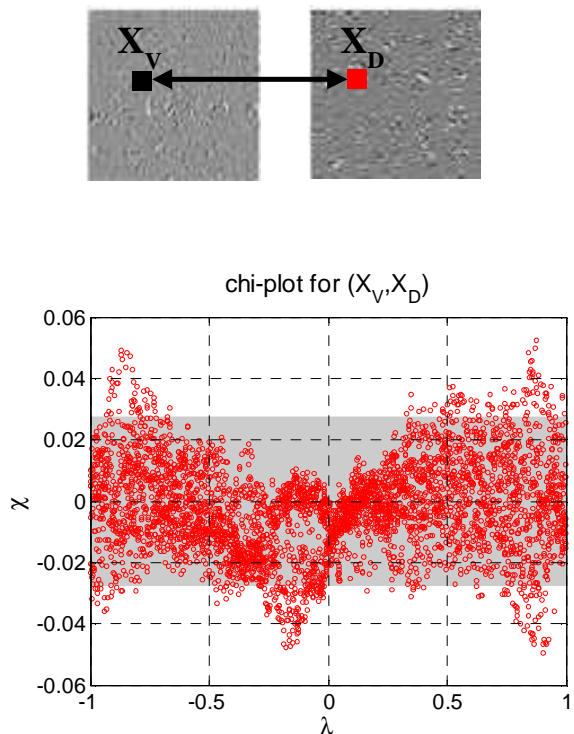

Dependence between a wavelet coefficient and its parent
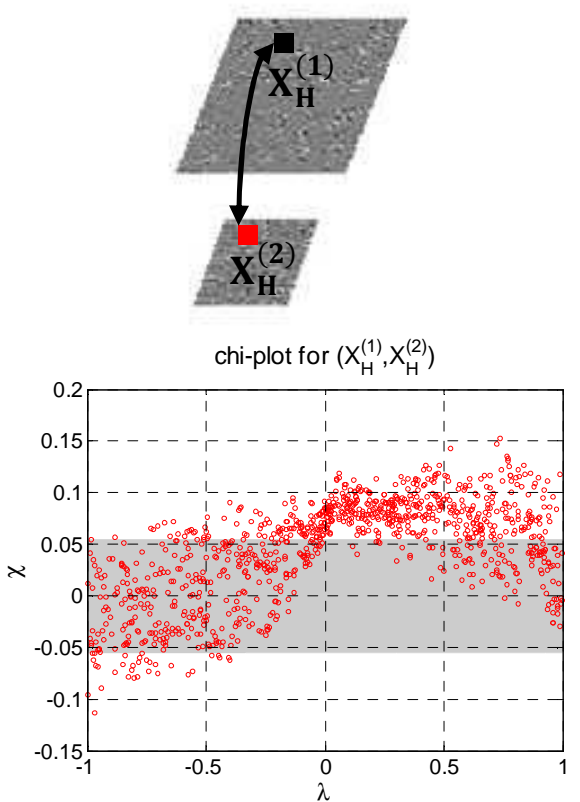

Fig. 1. Chi-plots to illustrate the different degrees of dependence between, intraband, interorientation and interscale, wavelet coefficient pairs.

Indeed, taking into account the interorientation and interscale independencies, we considered in this paper a wavelet subband $B_{k}, k=1, \ldots, K$ as a realization of a random vector $\vec{X}_{B_{k}}$ resulting from overlapping sliding windows (or another neighborhood geometry). The wavelet coefficient set is a realization of the overall random vector $\vec{X}=\left[\vec{X}_{B_{1}} ; \ldots ; \vec{X}_{B_{K}}\right]$. In this case, assuming all subbands are pairwise independent, the total JD between two joint distributions is the sum of the JD between the corresponding wavelet subbands PDFs

$$
J D\left(p_{1}\left(\vec{x} ; \theta_{1}\right), p_{2}\left(\vec{x} ; \theta_{2}\right)\right)=\sum_{k=1}^{K} J D\left(p_{1}\left(\vec{x}_{B_{k}} ; \theta_{k}^{(1)}\right), p_{2}\left(\vec{x}_{B_{k}} ; \theta_{k}^{(2)}\right)\right)
$$

where $\theta_{1}=\left\{\theta_{k}^{(1)}\right\}_{1, \ldots, K}$ and $\theta_{2}=\left\{\theta_{k}^{(2)}\right\}_{1, \ldots, K}$ are the hyperparameters of the two PDFs $p_{1}$ and $p_{2}$ respectively.

In the following, since this does not lead to confusion, each random vector $\vec{X}_{B_{k}}, k=1, \ldots, K$ will be noted $\vec{X}$ without specifying the subband index.

\section{GAUSSIAN COPULA BASED MULTIVARIATE MODELS}

In this section we establish stochastic multivariate modeling. Two families of Gaussian copula-based multivariate PDFs are considered, incorporating the intrasubband dependency while keeping a good fit to marginal distributions. As mentioned above, independency between subbands in orientations and scales is assumed while intrasubband dependences are exploited. The proposed approach characterizes statistical interactions within local neighborhoods.

After image decomposition into subbands at multiple scales and orientations, neighbors around a reference coefficient are 


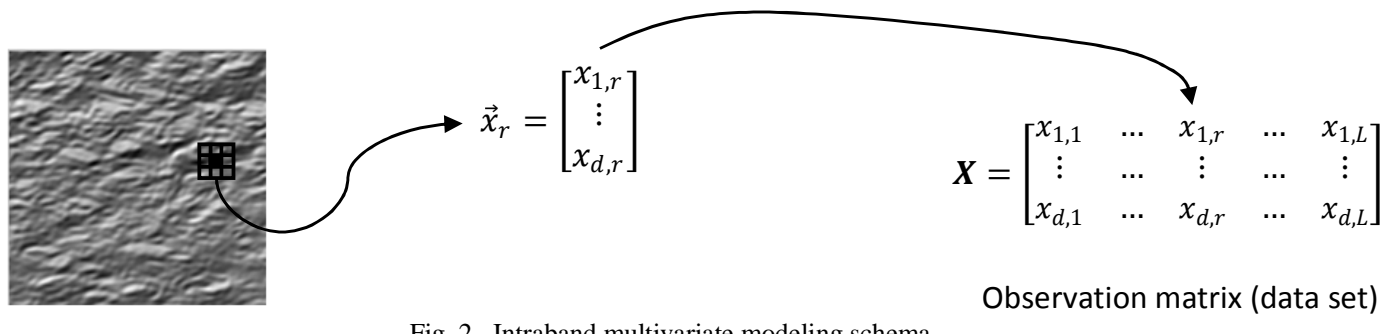

Fig. 2. Intraband multivariate modeling schema

gathered into a $d$-dimensional column vector $\vec{X}=\left(x_{1}, \ldots, x_{d}\right)^{t}$ where $d$ is the neighborhood size. Under the spatial homogeneity assumption of each subband, multiple observations of the vector $\vec{X}$ are obtained by moving a window across a subband and the samples of the $d$-dimensional vector $\vec{X}$ are noted $\vec{x}_{r}=\left(x_{1, r}, \ldots, x_{d, r}\right)^{t}, r=1, \ldots, L$ where $L$ is the size of a wavelet subband.

As shown in Fig. 2 , the data set to be modeled is then reorganized into the observation matrix

$$
\boldsymbol{X}=\left[\vec{x}_{1}, \ldots, \vec{x}_{r}, \ldots, \vec{x}_{L}\right]
$$

\section{A. A brief review of copulas}

Copulas have become a popular multivariate modeling tool in many fields such as finance, biomedical studies or hydrological modeling, where multivariate dependence is of interest [42]-[44]. The concept of copula relies on the desirable property of separating the study of marginal distributions from that of dependence. Indeed, the dependence between components is entirely embedded in the copula, so it provides a simple description of the dependence structure independently of the marginals.

This section recalls a few basic definitions that will be useful for the remainder of the paper. For a rigorous mathematical presentation of the concept of copulas, see [45]. A copula $C$ is a joint cumulative distribution function (CDF) defined on the $d$ dimensional unit cube $[0,1]^{\mathrm{d}}$ such that every marginal is uniform on $[0,1]$. The fact that the copula can be very useful for representing multivariate distributions with arbitrary marginals comes from the following result. Sklar's theorem [46] states that given a $d$-dimensional random vector $\overrightarrow{\mathrm{X}}=\left(x_{1}, \ldots, x_{\mathrm{d}}\right)^{\mathrm{t}}$ with continuous marginal CDFs $\mathrm{F}_{1}, \ldots, \mathrm{F}_{\mathrm{d}}$, there exists a unique copula $C$ such that

$$
F\left(x_{1}, \ldots, x_{d}\right)=C\left(F_{1}\left(x_{1}\right), \ldots, F_{d}\left(x_{d}\right)\right) \forall x=\left(x_{1}, \ldots, x_{d}\right) \in \mathbb{R}^{d}
$$

Conversely, if $C$ is a copula and $F_{1}, \ldots, F_{d}$ are CDFs then the function defined by $C\left(F_{1}\left(x_{1}\right), \ldots, F_{d}\left(x_{d}\right)\right)$ is a joint CDF with marginals $F_{1}, \ldots, F_{d}$. Moreover if the function $C$ is continuous and differentiable, then the copula density is given by

$$
c\left(u_{1}, \ldots, u_{d}\right)=\frac{\partial^{d} C\left(u_{1}, \ldots, u_{d}\right)}{\partial u_{1} \ldots \partial u_{d}}
$$

In this case, the joint probability density function of $\vec{X}$ can be written as

$$
f\left(x_{1}, \ldots, x_{d}\right)=c\left(F_{1}\left(x_{1}\right), \ldots, F_{d}\left(x_{d}\right)\right) \prod_{i=1}^{d} f_{i}\left(x_{i}\right)
$$


where $f_{i}, i=1, \ldots, d$ are the marginal PDFs. Thus, a joint multivariate PDF is uniquely defined given marginal PDFs and copula density.

Several copula families were previously proposed such as elliptic copulas, Student t copula or Archimedean copulas which differ in their dependence representation. For texture modeling, previous works have highlighted the key role played by the linear dependence, i.e. the correlation. Thus, in our work, among elliptic sub-family of copulas, we consider the Gaussian Copula [47] for three reasons:

- Gaussian Copula-based multivariate PDFs are a good fit to the statistics of wavelet coefficient subbands. They accurately capture both the marginal and joint distributions of wavelet subband coefficients.

- $\quad$ Related hyperparameters can be easily estimated using ML-based estimator (see sub-sections C and D).

- The existence of a closed form of KLD and consequently of JD between Gaussian Copula-based multivariate PDFs (see subsection E).

The Gaussian Copula is of practical interest since it can be easily implemented and its dependence structure is intuitive, based on correlation coefficients. The Gaussian Copula density is expressed by re-writing the multivariate normal density in the form given by equation (9) as follows

$$
c\left(u_{1}, \ldots, u_{d}\right)=\frac{1}{|\Sigma|^{1 / 2}} \exp \frac{-\vec{y}^{t}\left(\Sigma^{-1}-I\right) \vec{y}}{2}
$$

where $\vec{y}^{t}=\left(y_{1}, \ldots, y_{d}\right)$ stands for the transpose of vector $\vec{y}$ which is a vector of normal scores such that $y_{i}=\phi^{-1}\left(u_{i}\right)$, and $\phi$ for the $\mathrm{CDF}$ of the normalized Gaussian distribution $N(0,1)$. The matrix $I$ is the $d$-dimensional identity matrix and $\Sigma$ is the covariance matrix with ones in the diagonal.

\section{B. Gaussian Copula-based multivariate modeling estimation}

Let $\eta=\left(\eta_{1}, \ldots, \eta_{d}\right)$ be the set of marginal parameters. The hyperparameters of the Gaussian Copula-based multivariate model to be estimated are $\theta=\{\eta, \Sigma\}$ where $\Sigma$ is the covariance matrix of a Gaussian distribution.

The ML estimator of the hyperparameters associated to the joint $\operatorname{PDF} f(\vec{x} ; \theta)$ is given by

$$
\hat{\theta}=\{\hat{\eta}, \hat{\Sigma}\}=\arg \max _{\eta, \Sigma} \log \prod_{r=1}^{L} f\left(\vec{x}_{r} ; \theta\right)
$$

It has been shown in [48] that the marginal parameters $\hat{\eta}=\left(\hat{\eta}_{1}, \ldots, \hat{\eta}_{d}\right)$ could be estimated separately from the covariance matrix $\Sigma$. This leads to a simple procedure to estimate the full set of unknown hyperparameters:

1. $\left(\eta_{1}, \ldots, \eta_{d}\right)$ are estimated by using the ML estimator of marginals independently:

$$
\hat{\eta}_{i}=\arg \max _{\eta_{i}} \log \prod_{r=1}^{L} f_{i}\left(x_{i, r} ; \eta_{i}\right)
$$


(a)

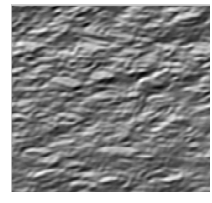

Initial subband (b)

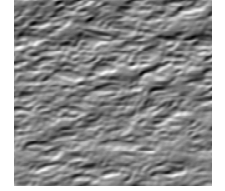

Gaussianized subband

Fig. 3. The transformed subband (b) is obtained from the initial one (a) by Gaussianization in order to estimate Gaussian Copula covariance matrix

2. Each observation $\vec{x}_{r}=\left(x_{1, r}, \ldots, x_{d, r}\right)^{t}$ from the observation matrix $\boldsymbol{X}$ (7) is transformed to $\vec{y}_{r}=\left(y_{1, r}, \ldots, y_{d, r}\right)^{t}$ by $y_{i, r}=$ $\phi^{-1}\left(F\left(x_{i, r} ; \eta_{i}\right)\right), r=1, \ldots, L$ and $i=1, \ldots, d$. The transformed data set is then reorganized into a matrix $\boldsymbol{G}=\left[\vec{y}_{1}, \ldots, \vec{y}_{L}\right]$ containing realizations of a Gaussian vector $\vec{y}=\left(y_{1}, \ldots, y_{d}\right)^{t}$. This transformation is a Gaussianization procedure of the subband. The Gaussianized subband is considered as a realization of a Gaussian vector $\vec{Y}$. An example of subband Gaussianization is illustrated in Fig. 3; each neighborhood $\vec{x}_{r}$ in the initial subband (a) is transformed to $\vec{y}_{r}$ which is an observation of a random Gaussian vector $\vec{Y}$. Hence, the subband resulted (b) is a realization of this Gaussian vector and said to be Gaussianized.

Finally, the ML estimate for $\Sigma$ is the sample covariance matrix of Gaussian observations $\vec{y}_{1}, \ldots, \vec{y}_{L}$ :

$$
\hat{\Sigma}=\frac{1}{\mathrm{~L}} \sum_{\mathrm{r}=1}^{\mathrm{L}} \vec{y}_{r} \vec{y}_{r}^{t}=\frac{1}{\mathrm{~L}} \boldsymbol{G} \boldsymbol{G}^{t}
$$

Since generalized Gaussian and Weibull distributions were successfully used to represent marginal distributions of wavelet coefficients and their magnitudes respectively [2], [9], we propose the Gaussian Copula-based Multivariate generalized Gaussian (GC-MGG) and the Gaussian Copula-based Multivariate Weibull (GC-MWbl) distributions for modeling the joint PDF of subband coefficients or their magnitude.

\section{Gaussian Copula-based Multivariate generalized Gaussian distribution GC-MGG}

Using Gaussian Copula density, we define the GC-MGG probability density function by

$$
\begin{gathered}
f_{G C-M G G}(\vec{x} ; \theta)=\frac{1}{|\Sigma|^{1 / 2}} \exp \frac{-\vec{y}^{t}\left(\Sigma^{-1}-I\right) \vec{y}}{2} \times\left(\frac{\beta}{2 \alpha \Gamma(1 / \beta)}\right)^{d} \exp -\sum_{i=1}^{d}\left(\frac{\left|x_{i}\right|}{\alpha}\right)^{\beta} \\
\forall \vec{x}=\left(x_{1}, \ldots, x_{d}\right) \in \mathbb{R}^{d}
\end{gathered}
$$

where $\theta=(\alpha, \beta, \Sigma)$ denotes the hyperparameters set, $\alpha>0, \beta>0$ are the scale and the shape parameter respectively, $\Sigma$ is the covariance matrix of the Gaussian vector $\vec{y}$ defined by $y_{i}=\phi^{-1}\left(F\left(x_{i} ; \alpha, \beta\right)\right)$.

$\phi$ is the CDF of the normal distribution $N(0,1)$ and $F(t ; \alpha, \beta)=\left\{\begin{array}{c}0.5\left(1+\gamma\left((t / \alpha)^{\beta}, 1 / \beta\right)\right) t \geq 0 \\ 1-F(-t) t<0\end{array}\right.$ is the generalized Gaussian CDF, where $\Gamma(z)=\int_{0}^{\infty} e^{-t} t^{z-1} d t$ is the Gamma function and $\gamma(y, z)=\frac{1}{\Gamma(z)} \int_{0}^{y} e^{-t} t^{z-1} d t$ is the incomplete Gamma function [49]. 
Note that the definition of GC-MGG (11) includes the particular case of the multivariate Gaussian density for $\beta=2$ and of the generalized Gaussian density when $d=1$.

The ML estimate of marginal parameters can be found for example in [2] or [50]. By construction, due to the use of a sliding overlapping window to construct the observation matrix $\boldsymbol{X}$ (7), all the marginals have the same parameters $(\alpha, \beta)$. Hence, we can use any observation $x_{i}=\left(x_{i, 1}, \ldots, x_{i, L}\right)$ which contains all the coefficients of a wavelet subband to estimate $(\alpha, \beta)$.

Fig. 4 and Fig. 5 show examples of bivariate and trivariate empirical PDFs of intraband wavelet coefficients compared to the estimated GC-MGG densities. When empirical joint densities exhibit a striking non-Gaussian behavior, the fitted GC-MGG densities can characterize this behavior. The fits are quite good and support the use of Gaussian Copula-based densities for modeling wavelet coefficients. The third column (Fig. 4) corresponds to a special case. The two coefficients are Gaussian and decorrelated as shown by the empirical PDF (dotted contours are nearly circular); this behavior is confirmed by the hyperparameters of the fitted GC-MGG: the shape parameter is near 2 which characterizes a Gaussian marginal and the covariance matrix is close to the identity matrix which implies independence.

\section{Gaussian Copula-based Multivariate Weibull distribution GC-MWbl}

We generalize the novel univariate model introduced by Kwitt and Uhl in [8], [9] by defining the GC-MWbl to model the joint statistics of wavelet coefficients' magnitude. The GC-MWbl probability density function is given by

$$
\begin{gathered}
f_{G C-M W b l}(\vec{x} ; \theta)=\frac{1}{|\Sigma|^{1 / 2}} \exp \frac{-\vec{u}^{t}\left(\Sigma^{-1}-I\right) \vec{u}}{2} \times\left(\frac{a}{b}\right)^{d} \frac{\prod_{i=1}^{d} x_{i}^{a-1}}{b^{d(a-1)}} \exp -\sum_{i=1}^{d}\left(\frac{x_{i}}{b}\right)^{a} \\
\forall \vec{x}=\left(x_{1}, \ldots, x_{d}\right) \in\left(\mathbb{R}^{+}\right)^{d}
\end{gathered}
$$

where $\theta=(a, b, \Sigma)$ denotes the hyperparameters set, $a>0, b>0$ are the shape and scale parameter respectively, $\Sigma$ is the covariance matrix of the Gaussian vector $\vec{u}$ vector defined by $u_{i}=\phi^{-1}\left(F\left(x_{i} ; a, b\right)\right) . \phi$ is the CDF of the normal distribution $N(0,1)$ and $F(t ; a, b)=1-e^{-(t / b)^{a}} \forall t \geq 0$.

The ML estimator of $(a, b)$ can be found in [8] or [50] and $\Sigma$ is estimated as indicated in part B. 


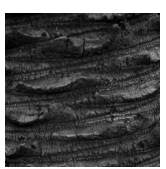

Bark.0000

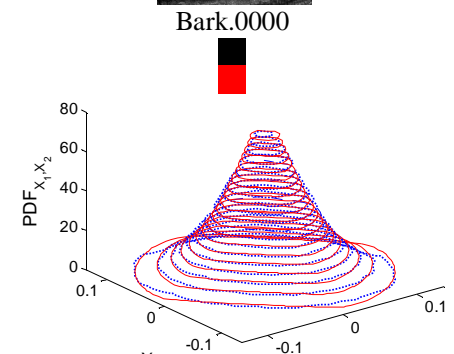

$\mathrm{X}_{2}^{-0.1}-0.1 \quad \mathrm{X}$

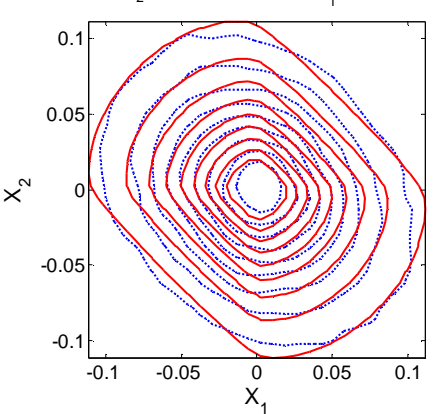

$\alpha=0.0171, \beta=1.2776$,

$\Sigma=\left[\begin{array}{cc}1 & -0.2084 \\ -0.2084 & 1\end{array}\right]$

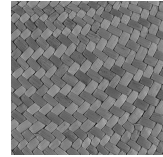

Fabric.0000
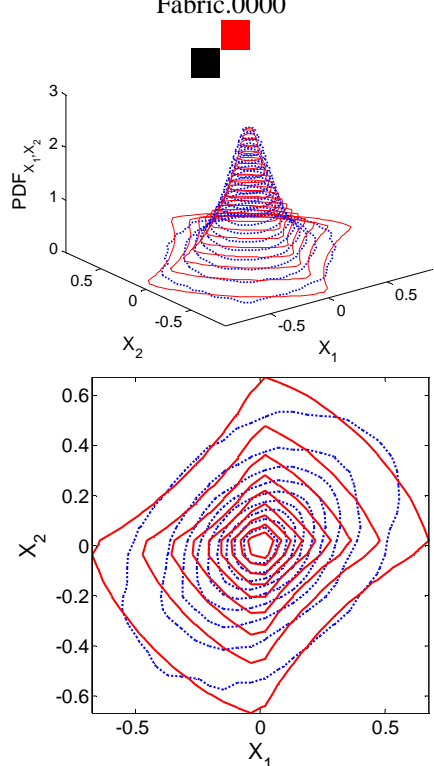

$\alpha=0.2921, \beta=0.9562$,

$\Sigma=\left[\begin{array}{cc}1 & 0.3937 \\ 0.3937 & 1\end{array}\right]$

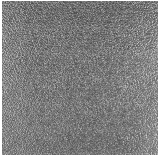

Metal.0000

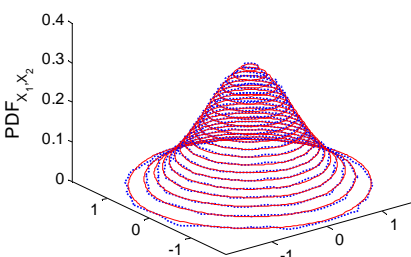

$\mathrm{x}_{2} \mathrm{x}^{-1} \mathrm{x}_{1}$

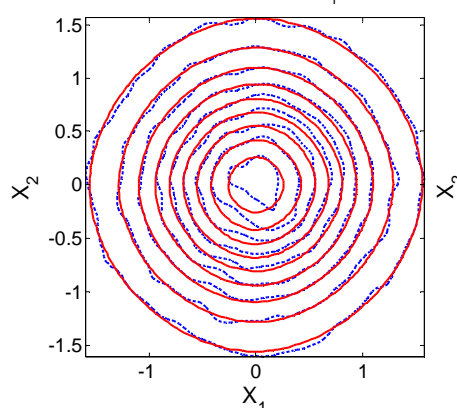

$\alpha=1.0025, \beta=1.8652$,

$\Sigma=\left[\begin{array}{cc}1 & 0.0061 \\ 0.0061 & 1\end{array}\right]$

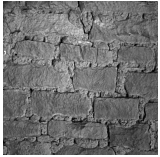

Brick.0005

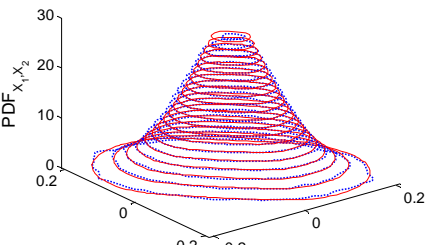

$\mathrm{X}_{2} \quad-0.2 \quad-0.2 \quad \mathrm{X}_{1}$

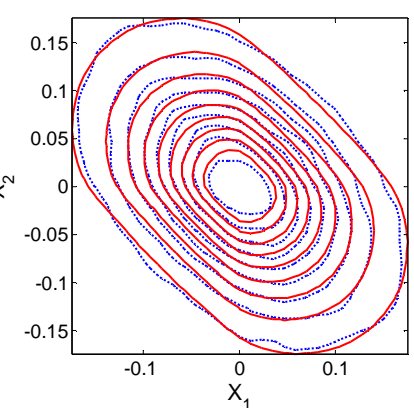

$\alpha=0.1023, \beta=1.5309$,

$\Sigma=\left[\begin{array}{cc}1 & -0.4840 \\ -0.4840 & 1\end{array}\right]$

Fig. 4. Wavelet subband coefficient bivariate empirical PDF (dotted lines) fitted with a GC-MGG density (solid lines). First row: four texture images from VisTex database. Second row: the neighborhood geometry considered to estimate the bivariate empirical PDF and GC-MGG density. Third row: the fitting of the bivariate empirical PDF with a GC-MGG density in a 3D view. Fourth row: the fitting of the bivariate empirical PDF with a GC-MGG density in a 2D view using isolines. Fifth row: the hyperparameters of the estimated GC-MGG density. The wavelet subbands considered are from left to right horizontal, diagonal, diagonal, and horizontal at the first scale using Daubechies' filter 'db4'.
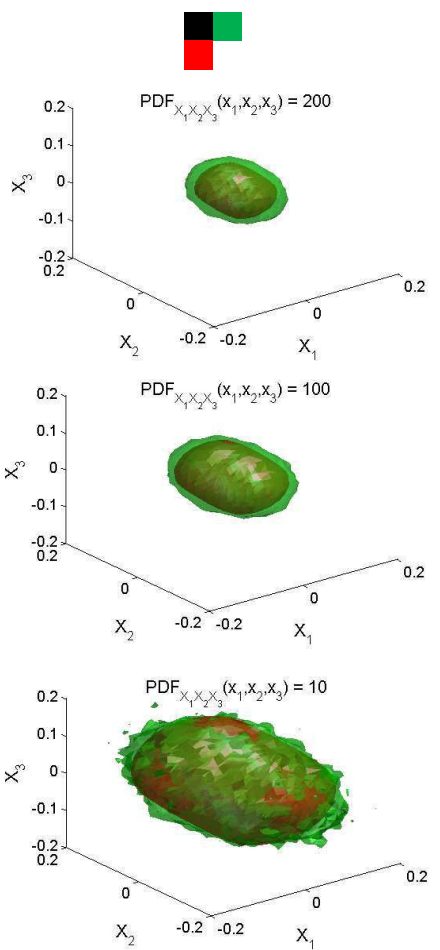

$\Sigma=\left[\begin{array}{ccc}\alpha=0.0171, \beta=1.2776 \\ 1 & -0.2084 & -0.2153 \\ -0.2084 & 1 & 0.0803 \\ -0.2153 & 0.0803 & 1\end{array}\right]$
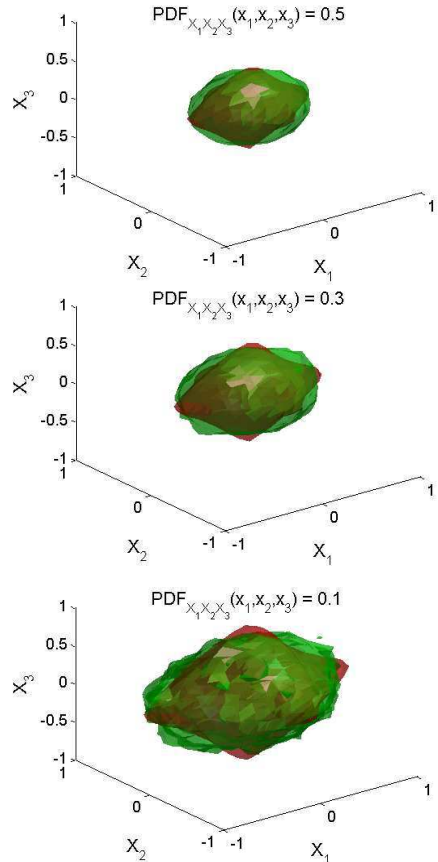

$\alpha=0.2921, \beta=0.9562$ $\Sigma=\left[\begin{array}{ccc}1 & 0.3937 & -0.4134 \\ 0.3937 & 1 & -0.6157 \\ -0.4134 & -0.6157 & 1\end{array}\right]$
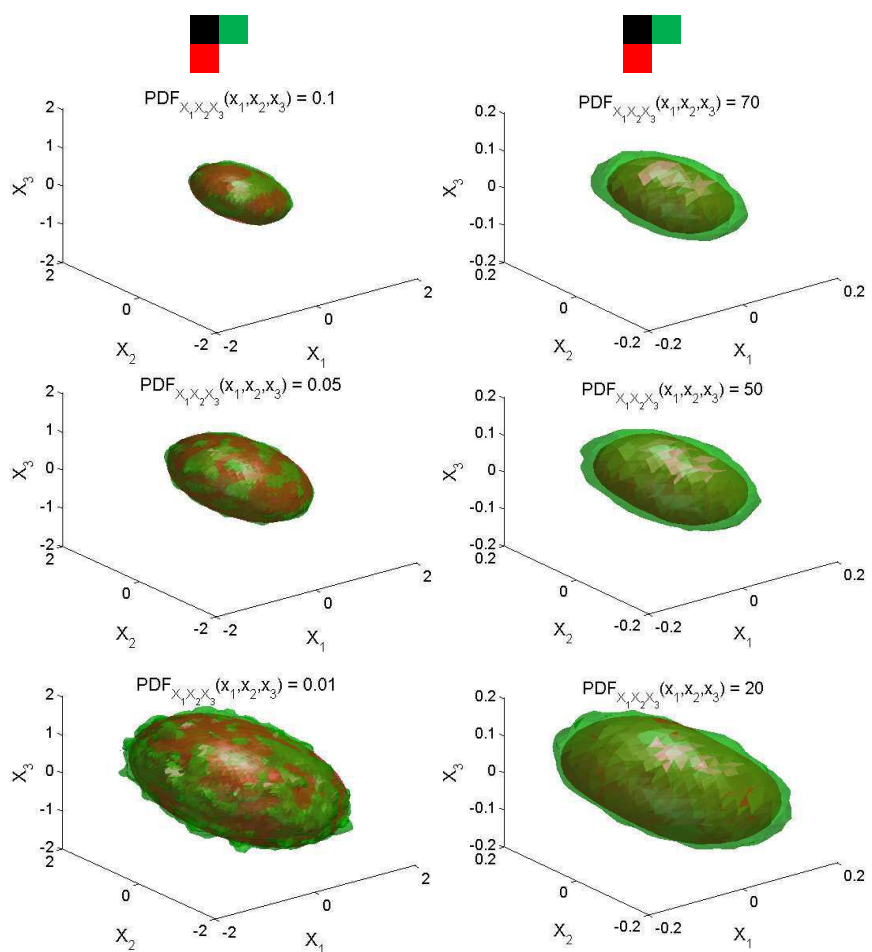

$\alpha=1.0025, \beta=1.8652, \quad \alpha=0$ $\alpha=0.1023, \beta=1.5309$

$=\left[\begin{array}{ccc}1 & -0.4862 & 0.0061 \\ -0.4862 & 1 & -0.0091 \\ 0.0061 & -0.0091 & 1\end{array}\right] \Sigma=\left[\begin{array}{ccc}1 & -0.4840 & -0.4366 \\ -0.4840 & 1 & 0.2944 \\ -0.4366 & 0.2944 & 1\end{array}\right]$

Fig. 5. Wavelet subband coefficient trivariate empirical PDF (green surfaces) fitted with a GC-MGG density (red surfaces). First row: the neighborhood geometry considered to estimate the trivariate empirical PDF and GC-MGG density. Second to Fourth row: the fitting of the trivariate empirical PDF with a GC-MGG density in a $3 \mathrm{D}$ view using isosurfaces. The images, subbands, and wavelet filter used are the same as in Fig. 4. 


\section{E. Similarity Measurement between Gaussian Copula Based Multivariate Models}

In this work, the similarity between two images is measured by comparing the sets of texture features extracted from wavelet decomposition. We use Gaussian Copula-based multivariate models (GC-MGG and GC-MWbl) to represent multidimensional histograms of subband coefficients or their magnitudes.

Given two Gaussian Copula-based multivariate models $\left\{\begin{array}{l}f\left(\vec{x} ; \theta_{1}\right)=c\left(F_{1}\left(x_{1}\right), \ldots, F_{d}\left(x_{d}\right)\right) \prod_{i=1}^{d} f_{i}\left(x_{i}\right) \\ g\left(\vec{x} ; \theta_{2}\right)=c\left(G_{1}\left(x_{1}\right), \ldots, G_{d}\left(x_{d}\right)\right) \prod_{i=1}^{d} g_{i}\left(x_{i}\right)\end{array}\right.$ we note $\theta_{1}=\left\{\left(\eta_{1}^{(1)}, \ldots, \eta_{d}^{(1)}\right), \Sigma_{1}\right\}$ and $\theta_{2}=\left\{\left(\eta_{1}^{(2)}, \ldots, \eta_{d}^{(2)}\right), \Sigma_{2}\right\}$ the hyperparameters of $f$ and $g$ respectively.

We measure the similarity using JD which is the symmetrized version of KLD. In order to compute $J D\left(f\left(\vec{x} ; \theta_{1}\right) \| g\left(\vec{x} ; \theta_{2}\right)\right)$ we first derive the general formula for KLD between two Gaussian Copula-based multivariate models given as (for more details, please see the Appendix)

$$
\begin{gathered}
K L D\left(f\left(\vec{x} ; \theta_{1}\right) \| g\left(\vec{x} ; \theta_{2}\right)\right)=\int \ldots \int f\left(\vec{x} ; \theta_{1}\right) \log \frac{f\left(\vec{x} ; \theta_{1}\right)}{g\left(\vec{x} ; \theta_{2}\right)} d \vec{x} \\
=\sum_{i=1}^{d} K L D\left(f_{i}\left(x_{i} ; \eta_{i}^{(1)}\right) \| g_{i}\left(x_{i} ; \eta_{i}^{(2)}\right)\right)+0.5\left(\operatorname{tr}\left(\Sigma_{2}^{-1} \Sigma_{1}\right)+\log \frac{\left|\Sigma_{2}\right|}{\left|\Sigma_{1}\right|}-d\right)
\end{gathered}
$$

Hence, the closed form of JD is

$$
\begin{gathered}
J D\left(f\left(\vec{x} ; \theta_{1}\right) \| g\left(\vec{x} ; \theta_{2}\right)\right)=K L D\left(f\left(\vec{x} ; \theta_{1}\right) \| g\left(\vec{x} ; \theta_{2}\right)\right)+K L D\left(g\left(\vec{x} ; \theta_{2}\right) \| f\left(\vec{x} ; \theta_{1}\right)\right) \\
=\sum_{i=1}^{d} J D\left(f_{i}\left(x_{i} ; \eta_{i}^{(1)}\right) \| g_{i}\left(x_{i} ; \eta_{i}^{(2)}\right)\right)+0.5\left(\operatorname{tr}\left(\Sigma_{2}^{-1} \Sigma_{1}\right)+\operatorname{tr}\left(\Sigma_{1}^{-1} \Sigma_{2}\right)\right)-d
\end{gathered}
$$

Finally, if we denote $\theta_{k}^{(1)}$ and $\theta_{k}^{(2)}, k=1, \ldots, K$ the wavelet subband hyperparameters estimated from two images $I_{1}$ and $I_{2}$ respectively, then the overall similarity measure is the sum of JD across all subbands:

$$
D\left(I_{1}, I_{2}\right)=\sum_{k=1}^{K} J D\left(f\left(\vec{x} ; \theta_{k}^{(1)}\right) \| g\left(\vec{x} ; \theta_{k}^{(2)}\right)\right)
$$

In the case of the two proposed multivariate models GC-MGG and GC-MWbl, the JDs between two joint PDFs are

$$
\begin{gathered}
J D\left(f_{G C-M G G}\left(\vec{x} ; \alpha_{1}, \beta_{1}, \Sigma_{1}\right) \| g_{G C-M G G}\left(\vec{x} ; \alpha_{2}, \beta_{2}, \Sigma_{2}\right)\right) \\
=\mathrm{d}\left(\left(\frac{\alpha_{1}}{\alpha_{2}}\right)^{\beta_{2}} \frac{\Gamma\left(\frac{\beta_{2}+1}{\beta_{1}}\right)}{\Gamma\left(1 / \beta_{1}\right)}-\frac{1}{\beta_{1}}+\left(\frac{\alpha_{2}}{\alpha_{1}}\right)^{\beta_{1}} \frac{\Gamma\left(\frac{\beta_{1}+1}{\beta_{2}}\right)}{\Gamma\left(1 / \beta_{2}\right)}-\frac{1}{\beta_{2}}\right)+0.5\left(\operatorname{tr}\left(\Sigma_{2}^{-1} \Sigma_{1}\right)+\operatorname{tr}\left(\Sigma_{1}^{-1} \Sigma_{2}\right)\right)-d
\end{gathered}
$$

and

$$
\begin{gathered}
J D\left(f_{G C-M W b l}\left(\vec{x} ; a_{1}, b_{1}, \Sigma_{1}\right) \| g_{G C-M W b l}\left(\vec{x} ; a_{2}, b_{2}, \Sigma_{2}\right)\right)=\mathrm{d}\left(\Gamma\left(\frac{\mathrm{a}_{2}}{\mathrm{a}_{1}}+1\right)\left(\frac{\mathrm{b}_{1}}{\mathrm{~b}_{2}}\right)^{\mathrm{a}_{2}}+\Gamma\left(\frac{\mathrm{a}_{1}}{\mathrm{a}_{2}}+1\right)\left(\frac{\mathrm{b}_{2}}{\mathrm{~b}_{1}}\right)^{\mathrm{a}_{1}}\right. \\
\left.+\left(\mathrm{a}_{1}-\mathrm{a}_{2}\right) \ln \left(\frac{\mathrm{b}_{1}}{\mathrm{~b}_{2}}\right)+\gamma\left(\frac{\mathrm{a}_{2}}{\mathrm{a}_{1}}+\frac{\mathrm{a}_{1}}{\mathrm{a}_{2}}-2\right)-2\right)+0.5\left(\operatorname{tr}\left(\Sigma_{2}^{-1} \Sigma_{1}\right)+\operatorname{tr}\left(\Sigma_{1}^{-1} \Sigma_{2}\right)\right)-d
\end{gathered}
$$

where $\gamma=-\Psi(1) \simeq 0.57721$ denotes Euler-Mascheroni constant. 


\section{EXPERIMENTAL RESULTS}

\section{A. Experimental Setting}

In this section experiments are conducted to evaluate the performance of multivariate modeling in a retrieval framework using wavelet transforms. A comparison is made between the proposed Gaussian Copula-based models (GC-MGG and GC-MWbl), and previous works including the univariate models presented in [2], [9] (GG and Wbl) and, Mixture Gaussian model (MGMix) of [27]-[29], multivariate Gaussian (MG) model used by [20] and the Multivariate generalized Gaussian (MGG) model introduced in [32].

In our simulations, three experimental benchmarks are considered to evaluate the retrieval performance:

- EB1: 40 grayscale texture classes from the MIT Vision Texture Database (VisTex) [51] are addressed. This experimental setup is conventional and thus is largely used in the literature devoted to texture image retrieval issue [2], [9], [35]. From each of these texture images of size $512 \times 512$ pixels, 16 non-overlapping subimages $(128 \times 128$ pixels) are created in order to form 16 samples of each texture class. A test database of 640 texture images is then obtained.

- $E B 2$ : from the entire Brodatz texture album [52], $N$ texture classes are randomly selected, with increasing values of $(N=$ $10,15,20,25,30,35,40)$. For each value of $N$, we take 25 random subsets of $N$ texture classes from the album. In a random subset, each of the $N$ texture images of size $640 \times 640$ pixels is then divided into sixteen 160x160 non-overlapping subimages creating a test database of $N \times 16$ texture samples. In summary, the second benchmark is composed of $25 \times 7=175$ random test databases of different sizes $N_{t}=N \times 16(N=10,15,20,25,30,35,40)$ while the classic one is composed from one test database of 640 samples.

- EB3: 250 texture classes from Amsterdam Library of Textures (ALOT) [53], and 476 texture classes from the novel texture image database Salzburg Textures (STex) [54] are considered. In the case of ALOT database, we select the grayscale version under the C1L1 capture condition. The STex database consists of true color texture images, hence we transform all images to grayscale ones. As for $E B 1$ and $E B 2$, Each image class is split into 16 non-overlapping subimages and two large test databases of 4000 and 7616 images are created.

We employed the orthogonal wavelet transform (OWT) with Daubechies' filters (db4 and db5) [55] and the Kinkgsbury's QShift $(14,14)$-tap filters in combination with $(13,19)$-tap near-orthogonal filters for Dual Tree Complex Wavelet Transform (DTCWT) [56]. We consider a square neighborhood of size $2 \times 2$ to construct observation matrices of intraband coefficients. Although we could consider a bigger neighborhood, it does not make relevant differences in accuracy retrieval as we will show at the subsection $\mathrm{F}$ of this part. A maximum of three scales of decomposition is chosen. Our experiments agree with [2] in that the size of the smallest subband resulting from a transform should be more than $16 x 16$ to ensure the consistency of PDF hyperparameter 
estimation.

For similarity measurement, JD is used which is the symmetrized version of KLD. The closed form of KLDs for GG and Wbl are derived in [2] and [8] respectively. In the case of MGmix no closed form expression of KLD exists, so the variational method introduced in [31] as an analytic approximation to KLD for MGmix models is considered.

In each retrieval experiment a query image is any one from the test database of size $N_{t}$. The relevant images for each query are the other 15 subimages obtained from the same image class. The number of correctly retrieved images (relevant images for a query image) is determined among the $K$ retrieved ones, in this case $K$ represents the size of the query. In our experiments, $N_{R}=15$ is the number of relevant subimages in each class. For a query image, let $n_{q}(K)$ be the number of correctly retrieved images among the $K$ retrieved ones. The quantitative evaluation consists in computing the three measures [57]:

- The retrieval recall with respect to a query $q$, is the ratio of the number of relevant images retrieved over the total number of relevant images in the database for the respective query. The average retrieval recall is then given for $K=1 \ldots N_{t}$ by

$$
\begin{aligned}
R(K) & =\text { mean }\left(\frac{\text { number of relevant images retrieved }}{\text { number of relevant images }}\right) \\
& =\frac{\sum_{q=1}^{N_{t}} n_{q}(K)}{N_{t} \times N_{R}}
\end{aligned}
$$

- The retrieval precision with respect to a query $q$, is the ratio of the number of relevant images retrieved over the number of total retrieved images. The average retrieval precision is then given for $K=1 \ldots N_{t}$ by

$$
\begin{aligned}
P(K) & =\text { mean }\left(\frac{\text { number of relevant images retrieved }}{\text { number of retrieved images }}\right) \\
& =\frac{\sum_{q=1}^{N_{t}} n_{q}(K)}{N_{t} \times K}
\end{aligned}
$$

- $\quad$ The average retrieval rate (ARR)

$$
A R R=\frac{\sum_{q=1}^{N_{t}} n_{q}\left(N_{R}\right)}{N_{t} \times N_{R}}
$$

In information retrieval literature, the recall/precision curve is commonly used to evaluate the performance of retrieval systems [58]. A method with recall/precision curve which will be on above of another is considered better and more suitable. The ARR is the average retrieval precision $P\left(N_{R}\right)$ after retrieving a number of images equal to the number of relevant images. High values of ARR denote a high retrieval rate.

\section{B. Retrieval Performance with EB1: univariate models vs Gaussian Copula-based multivariate models}

Table I summarizes the ARRs obtained by using GC-MGG and GC-MWbl over the corresponding univariate models GG and Wbl respectively. We can observe from the Table I that: 
TABLE I

AVERAGE RETRIEVAL RATES (\%) IN THE TOP 16 MATCHES USING ORTHOGONAL WAVELET TRANSFORM WITH DAUBECHIES' FILTER db4 AND DUAL TREE COMPLEX WAVELET TRANSFORM ON EB1 (VISTEX)

\begin{tabular}{|c|c|c|c|c|}
\hline \multirow{2}{*}{ Type of Transform } & \multicolumn{4}{|c|}{ Models } \\
\cline { 2 - 5 } & GG & Wbl & GC-MGG & GC-MWbl \\
\hline 1 scale & & & & \\
OWT, db4 & 70.52 & 69.37 & $\mathbf{7 9 . 7 8}$ & 75.81 \\
DT-CWT & 72.89 & 73.17 & $\mathbf{8 1 . 6 6}$ & 77.59 \\
\hline 2 scales & & & & \\
OWT, db4 & 76.42 & 75.92 & $\mathbf{8 1 . 9 4}$ & 79.61 \\
DT-CWT & 78.74 & 79.63 & $\mathbf{8 3 . 7 0}$ & 82.36 \\
\hline 3 scales & & & & \\
OWT, db4 & 78.78 & 78.27 & $\mathbf{8 3 . 1 9}$ & 81.55 \\
DT-CWT & 80.36 & 82.05 & $\mathbf{8 4 . 3 3}$ & $\mathbf{8 4 . 4 1}$ \\
\hline
\end{tabular}

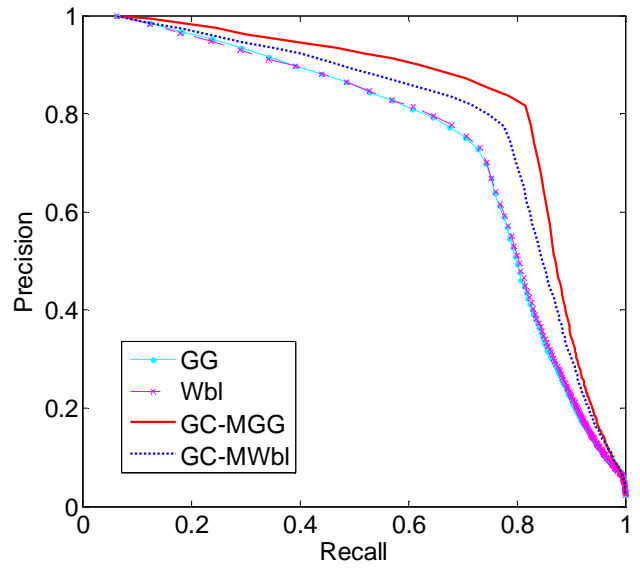

Fig. 6. Recall-Precision curves on EBI using DT-CWT with 1 decomposition level for GG, Wbl, GC-MGG and GC-MWbl.

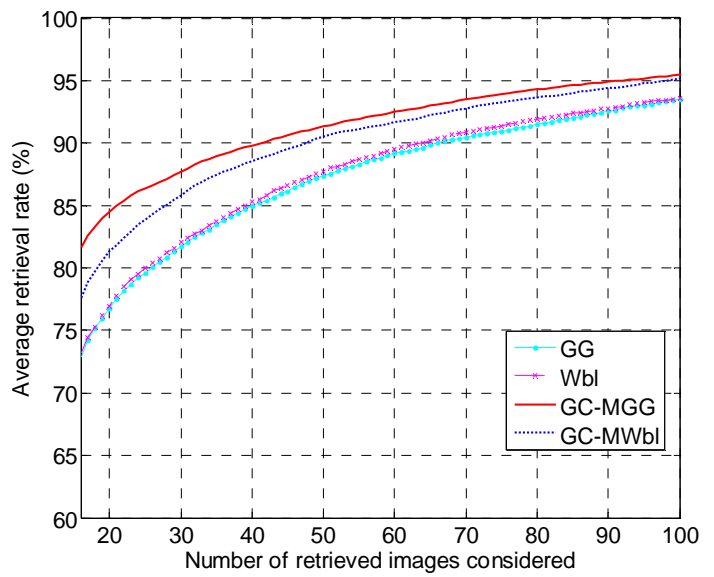

Fig. 7. Retrieval effectiveness according to the number of samples retrieved on $E B 1$ using DT-CWT with 1 decomposition level for GG, Wbl, GC-MGG and GC-MWbl.

1) Using Gaussian Copula-based models leads to improvement in retrieval accuracy. GC-MGG and GC-MWbl perform better than GG and $\mathrm{Wbl}$ by around $6 \%$ and $4 \%$ respectively. Even though GG and Wbl give equivalent performances, GC-MGG is slightly more efficient and improves retrieval performance by about $2 \%$ compared to GC-MWbl. This may be explained by the fact that GC-MGG modeling fits well the subband multidimensional histograms and especially in first decomposition level.

2) The improvement from one to two scales is more important for univariate models GG and Wbl (around 6\%) than for multivariate models GC-MGG and GC-MWbl (around 3\%). As reported in [2] and [9], increasing decomposition level does not provide further improvement. In addition, using four decomposition scales in our case leads to wavelet subbands of size 
8x8 for $E B 1$ and $E B 3$ or $10 \times 10$ for $E B 2$ so the estimate of covariance matrices becomes inaccurate.

3) There is a slight improvement using DT-CWT beside OWT (around 2\%). This observation is coherent with the results obtained in [8], [9]. This can be explained by the fact that six oriented subbands are provided by DT-CWT for each scale while the OWT provides only three. Hence, the size of signatures for DT-CWT is twofold compared to OWT. However it is interesting to observe that the two univariate models GG and $\mathrm{Wbl}$ are almost equivalent when one uses the same wavelet transform for both of them to extract signatures. In [8], [9] Kwitt and Uhl suggest the use of Wbl as an alternative to GG and they employ Wbl with DT-CWT while they use GG with OWT. We emphasize the fact that models must be used with the same transform tools to have a fair comparison of performance.

Fig. 6 depicts the recall/precision curves for the four different models. These results support the remarks mentioned above: the two univariate models, i.e. GG and Wbl, are equivalent while the use of Gaussian Copula-based models GC-MGG and GCMWbl improves significantly the retrieval accuracy. Fig. 7 compares the ARRs as a function of a query size $K$. This plot leads to a receiver operating characteristic (ROC) curves for each model. We can observe that when $K=42$ GC-MGG approach retrieves $90 \%$ of relevant texture samples from $E B 1$ while $K=48,64,66$ are required in order to retrieve the same percentage using GC-MWbl, GG and Wbl respectively. Using GC-MGG and GC-MWbl models, 95\% of relevant samples can be obtained when we retrieve 100 ones while the ARR still not reach this level of percentage for the two univariate models, i.e. GG and Wbl.

\section{Retrieval Performance with EB2: univariate models vs Gaussian Copula-based multivariate models}

The benchmark $E B 2$ is random and thus more objective than the conventional $E B 1$ for providing additional justification to use Gaussian Copula-based models. Table II summarizes the ARRs using different numbers of classes for the four compared models. We can observe an important improvement in retrieval rates for all the numbers of classes randomly selected when Gaussian Copula-based models are used. The improvement is around 6\% for GG versus GC-MGG and about $4 \%$ from Wbl to GC-MWbl. Thus, we can conclude that univariate models GG and Wbl are almost equivalent while using Gaussian Copula-based models GC-MGG and GC-MWbl increase significantly the retrieval rates.

\section{Retrieval Performance with EB3: univariate models vs Gaussian Copula-based multivariate models on large databases}

In Table III we summarize the ARR for the two large databases STex and ALOT. The GC-MGG and GC-MWbl models show again higher retrieval rates than GG and Wbl models. Nevertheless, the ARRs obtained on STex or ALOT (57.24\% and 43.25\% respectively) are unsatisfactory. These results are not really surprising; especially for STex database for which the color features appear very discriminant as shown in the work of Kwitt et al. [32]. We point out that we can improve retrieval rates for these databases if we take into account color information in addition to spatial dependency as it was shown in [59], but this is beyond the scope of our work where we are interested only in texture content of grayscale images. 
TABLE II

AVERAGE RETRIEVAL RATES (\%) ACCORDING TO THE NUMBER OF CLASSES CONSIDERED USING TWO LEVELS ORTHOGONAL WAVELET TRANSFORM WITH DAUBECHIES'

\begin{tabular}{|c|c|c|c|c|c|c|}
\hline Number of clasces & $\overline{G G}$ & & Improve & Whb & GC,MWhl & \\
\hline \multicolumn{7}{|l|}{$O W T, d b 4$} \\
\hline 40 & 71.39 & 79.16 & 7.77 & 71.49 & 77.29 & 5.80 \\
\hline 35 & 72.75 & 80.64 & 7.89 & 72.86 & 79.00 & 6.14 \\
\hline 30 & 73.62 & 81.29 & 7.67 & 73.74 & 79.41 & 5.68 \\
\hline 25 & 74.82 & 81.88 & 7.06 & 74.91 & 80.62 & 5.71 \\
\hline 20 & 77.51 & 84.23 & 6.72 & 77.60 & 82.80 & 5.21 \\
\hline 15 & 81.60 & 87.41 & 5.81 & 81.60 & 86.04 & 4.44 \\
\hline 10 & 81.83 & 86.91 & $\begin{array}{c}5.08 \\
\text { Mean }=6.86\end{array}$ & 81.85 & 85.50 & $\begin{array}{c}3.65 \\
\text { Mean }=5.23\end{array}$ \\
\hline \multicolumn{7}{|l|}{$D T-C W T$} \\
\hline 40 & 72.51 & 79.69 & 7.18 & 72.62 & 77.95 & 5.33 \\
\hline 35 & 73.94 & 81.24 & 7.3 & 74.05 & 79.89 & 5.84 \\
\hline 30 & 74.59 & 81.84 & 7.25 & 74.90 & 80.15 & 5.25 \\
\hline 25 & 75.77 & 82.46 & 6.69 & 76.22 & 81.29 & 5.07 \\
\hline 20 & 78.23 & 84.50 & 6.27 & 78.40 & 83.39 & 4.99 \\
\hline 15 & 82.48 & 87.63 & 5.15 & 82.86 & 86.67 & 3.81 \\
\hline \multirow[t]{2}{*}{10} & 82.16 & 86.99 & 4.83 & 82.40 & 85.80 & 3.40 \\
\hline & & & Mean $=6.38$ & & & Mean $=4.81$ \\
\hline
\end{tabular}

TABLE III

AVERAGE RETRIEVAL RATES (\%) IN THE TOP 16 MATCHES USING ORTHOGONAL WAVELET TRANSFORM WITH DAUBECHIES' FILTER db4 ON LARGE DATABASES OF EB3

\begin{tabular}{|c|c|c|c|c|c|c|c|c|}
\hline & & & & & & & & \\
\hline & GG & GC-MGG & Wbl & GC-MWbl & GG & GC-MGG & Wbl & GC-MWbl \\
\hline $\begin{array}{c}1 \text { scale } \\
\text { OWT,db4 } \\
\text { DT-CWT }\end{array}$ & $\begin{array}{l}31.64 \\
34.82\end{array}$ & $\begin{array}{l}43.91 \\
46.42\end{array}$ & $\begin{array}{l}31.05 \\
34.18\end{array}$ & $\begin{array}{l}37.60 \\
40.44\end{array}$ & $\begin{array}{l}23.68 \\
23.86\end{array}$ & $\begin{array}{l}30.02 \\
30.58\end{array}$ & $\begin{array}{l}23.42 \\
23.28\end{array}$ & $\begin{array}{l}27.01 \\
27.69\end{array}$ \\
\hline $\begin{array}{c}2 \text { scales } \\
\text { OWT,db4 } \\
\text { DT-CWT }\end{array}$ & $\begin{array}{l}40.70 \\
44.97 \\
\end{array}$ & $\begin{array}{l}50.11 \\
53.34 \\
\end{array}$ & $\begin{array}{l}40.18 \\
45.38 \\
\end{array}$ & $\begin{array}{l}44.70 \\
49.78 \\
\end{array}$ & $\begin{array}{l}31.99 \\
33.38 \\
\end{array}$ & $\begin{array}{l}36.44 \\
38.20 \\
\end{array}$ & $\begin{array}{l}31.55 \\
33.56 \\
\end{array}$ & $\begin{array}{l}34.52 \\
37.68 \\
\end{array}$ \\
\hline $\begin{array}{c}3 \text { scales } \\
\text { OWT,db4 } \\
\text { DT-CWT }\end{array}$ & $\begin{array}{r}45.89 \\
50.75 \\
\end{array}$ & $\begin{array}{l}\mathbf{5 3 . 8 1} \\
\mathbf{5 7 . 2 4} \\
\end{array}$ & $\begin{array}{r}45.69 \\
51.87 \\
\end{array}$ & $\begin{array}{l}50.11 \\
55.35 \\
\end{array}$ & $\begin{array}{l}37.86 \\
39.33 \\
\end{array}$ & $\begin{array}{r}41.86 \\
43.06 \\
\end{array}$ & $\begin{array}{l}37.39 \\
40.01 \\
\end{array}$ & $\begin{array}{l}40.14 \\
\mathbf{4 3 . 2 5} \\
\end{array}$ \\
\hline
\end{tabular}

\section{E. Retrieval Performance with EB1: other multivariate models vs Gaussian Copula-based multivariate models}

Here, the performance of the proposed Gaussian Copula-based models and three others multivariate models are compared: multivariate Gaussian (MG), Multivariate generalized Gaussian (MGG) and multivariate Gaussian mixture (MGmix). We use the Gaussianization method described in [20] to obtain Gaussianized wavelet subbands and hence we can model wavelet coefficients with MG model. The KLD and as a consequence the JD between MG models are widely used in literature, they can be found for instance in [60]. Approximated Rao geodesic distance is used as similarity measure between MGG distributions [32]. The MGmix model is fitted with 2, 3 and 4 components using the conventional Expectation maximization (EM) algorithm. Initial component parameters are randomly chosen and the EM algorithm is stopped after convergence or after 100 iterations. A small regularization number $\varepsilon=10^{-5}$ is added to the diagonal of covariance matrices to make them positive-definite. There is no closed form for KLD and JD between MGmix models but we can use an analytic approximation as mentioned in [27]. We have chosen the variational method to compute JD between MGmix models, because among all methods introduced in [31] it is the one which leads to the best retrieval rates.

Table IV summarizes the ARRs resulting from the use of the compared multivariate models on the conventional EBI with OWT combined to Daubechies' filter db5 and DT-CWT. As we can see, on the one hand, the proposed GC-MGG and GC-MWb1 


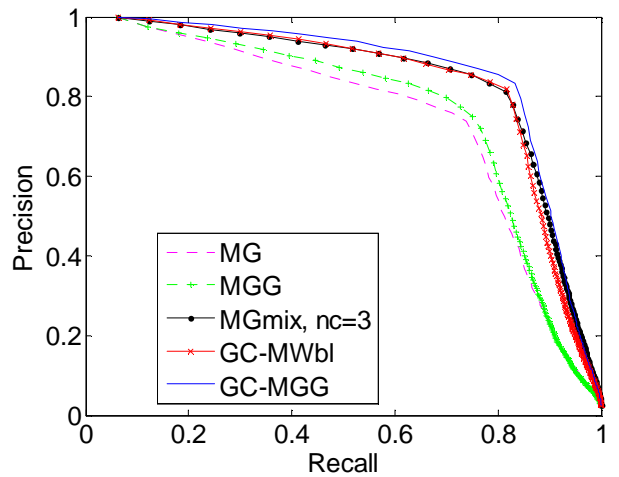

Fig. 8. Recall-Precision curves on EB1 using OWT, db5 with 3 decomposition levels for multivariate models.

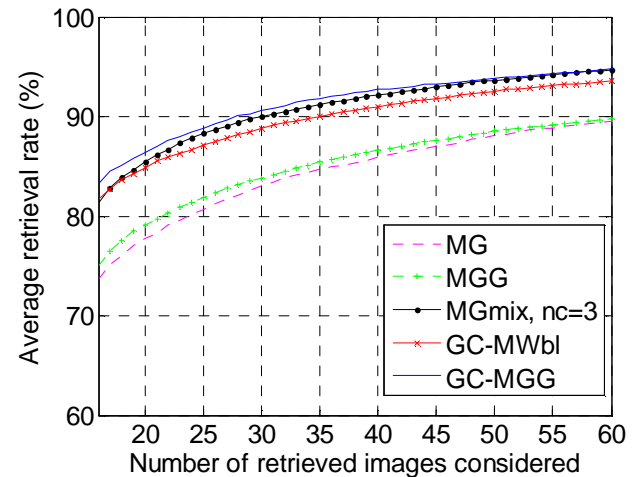

Fig. 9. Retrieval effectiveness according to the number of samples retrieved on EB1 using OWT, db5 with 3 decomposition levels for multivariate models.

TABLE IV

AVERAGE RETRIEVAL RATES (\%) FOR MULTIVARIATE MODELS IN THE TOP 16 MATCHES USING ORTHOGONAL WAVELET TRANSFORM WITH DAUBECHIES FILTER db5 AND DUAL TREE COMPLEX WAVELET TRANSFORM WITH EBI

\begin{tabular}{|c|c|c|c|c|c|c|c|}
\hline \multirow{2}{*}{ Type of Transform } & \multirow{2}{*}{ MG } & \multicolumn{3}{|c|}{ MGmix } & \multirow{2}{*}{ MGG } & \multirow{2}{*}{ GC-MGG } & \multirow{2}{*}{ GC-MWbl } \\
\hline & & $\mathrm{nc}=2$ & $\mathrm{nc}=3$ & $\mathrm{nc}=4$ & & & \\
\hline 1 scale & & & & & & & \\
\hline OWT, db5 & 62.38 & 71.79 & 73.12 & 75.18 & 64.16 & 79.57 & 75.18 \\
\hline DT-CWT & 65.71 & 78.19 & 79.90 & 80.05 & 71.51 & 81.66 & 77.59 \\
\hline \multicolumn{8}{|l|}{2 scales } \\
\hline OWT, db5 & 70.17 & 78.30 & 79.53 & 79.97 & 71.56 & 82.05 & 80.08 \\
\hline DT-CWT & 71.27 & 81.77 & 83.41 & 83.24 & 75.25 & 83.70 & 82.36 \\
\hline \multicolumn{8}{|l|}{3 scales } \\
\hline OWT, db5 & 73.74 & 81.06 & 81.41 & 80.07 & 75.06 & 83.31 & 81.75 \\
\hline DT-CWT & 74.59 & 83.82 & 84.04 & 83.07 & 78.08 & 84.33 & 84.41 \\
\hline
\end{tabular}

in most cases outperform MG, MGG and MGmix models. We remark that as for the previous experiments, there is an improvement in retrieval accuracy for all models when additional scales are used for wavelet decomposition. On the other hand, the best results are always obtained with DT-CWT compared to OWT. We note that adding mixture components for MGmix model does not improve significantly retrieval rates while it reduces the computation efficiency especially for similarity measure. For this reason, taking 2 or 3 components is a good trade-off for MGmix model to achieve competitive performance while respecting the constraint of computing time. We note that, although using computationally complex Gaussianization procedure of [20] to obtain Gaussian subbands in order to exploit MG model, the retrieval performance of MG model is less than these of univariate models. We point out that even if MGG model has achieved good performance when it was used with color texture images [32], its discrimination power decreases when it is employed to model only the spatial dependency of wavelet coefficients from grayscale texture images.

The same conclusions can be obtained from recall/precision curves (Fig. 8). Finally, ROC curves provide an additional justification of the improvement in retrieval framework using Gaussian Copula-based models (Fig. 9). For example, for GCMGG we need to use queries of size 28 to reach $90 \%$ of relevant samples while we must retrieve 30,36 and 60 images to reach the same percentage with MGmix, GC-MWbl, and MGG models respectively.

\section{F. Dimensionality trade-off}

We studied the effect of the neighborhood size and the number of decomposition levels on retrieval accuracy. We compared 
the ARRs using GC-MGG and MGmix models. The results are reported in Table V. We observe that the performance decreases for both models when using 4 scales. This substantiates the claim of Do and Vetterli [2] to use subbands with sufficient sizes to estimate model parameters. For instance, on EBI considering $128 \times 128$ images, the use of 4 scales leads to subbands with a smallest size $8 \times 8$ which is insufficient to estimate parameters regardless of the model used. With neighborhood of dimension $4 \times 4$ it is obvious that the estimation of covariance matrices of $16 \times 16$ size using data set of length 256 from a subband of size $16 \times 16$ will be inconsistent. Hence, the ARRs decrease when passing from 2 to 3 scales with neighborhood size bigger than $3 \times 3$. Unfortunately it is difficult to provide analytic relationship between neighborhood size (noted $d$ in Fig. 2) and the data set length (noted $L$ in Fig. 2) to have effective estimation neither for Gaussian Copula-based models nor for MGmix. However, data set must be large enough $(L \gg d)$ to properly estimate the model hyperparameters. We further observe that the expansion of neighborhood size does not significantly improve retrieval performance while this increases the JD computational complexity which depends on the dimension $d$ as it will be seen at the end of next part (Eq 17).

\section{G. Computational Complexity}

These experiments have been implemented in a Matlab R2011b environment on a Core 2 Duo (2.66 Ghz) PC with 3 GB of memory for tests on $E B 1$ and $E B 2$ and on a Core i7 (2.93 Ghz) with $8 \mathrm{~GB}$ for tests on $E B 3$. The comparative computational runtime is summarized in Table VI. To extract a signature of a 128x128 image, the runtime is less than 1 s for all compared models except for MGmix with more than 3 components and for MGG. Almost twofold time is required to compute GC-MGG signature compared to GG signature or GC-MWbl compared to Wbl, while 3 components MGmix and MGG require a computation time multiplied by 6 compared to GC-MGG. For example, the computational time to index EBI is about 96 seconds when GC-MGG is used whereas it is about 9 minutes for MGmix with 3 components. In a retrieval system the complexity of similarity measurement is crucial. The use of Gaussian Copula-based models needs 14 times more runtime to measure similarity than the use of univariate models, but it stays applicable even in case of large databases. For example, using GC-MGG or GCMWbl requires only 2.5 seconds to measure similarity between a 128x128 image and all the candidate images in test database of size 640 which is a reasonable runtime for a database of this size. We further observe that the use of ML similarity between Gaussian Copula-based models has a high computational complexity compared to the use of JD. If we use GC-MGG with ML and 1/8 subsampling to reduce data set, 19 seconds is needed to compute likelihood similarity between a query image and the 640 candidate images, while only 2.5 seconds are required if we use JD as similarity measure. According to the results of computational time, we note a relevant increase in retrieval accuracy using GC-MGG or GC-MWbl while keeping the use of these models tractable as in the case of univariate models GG and Wbl, and this is not the case when the same models are used with ML similarity measure. 
TABLE V

AVERAGE RETRIEVAL RATES (\%) USING DIFFERENT NEIGHBORHOOD SIZES AT DIFFERENT DECOMPOSITION LEVELS WITH DAUBECHIES' FILTER db4 ON EB 1

\begin{tabular}{|c|c|c|c|c|c|c|c|c|}
\hline & \multicolumn{2}{|c|}{1 scale } & \multicolumn{2}{|c|}{2 scales } & \multicolumn{2}{|c|}{3 scales } & \multicolumn{2}{|c|}{4 scales } \\
\hline Smallest subband size & \multicolumn{2}{|c|}{$64 \times 64$} & \multicolumn{2}{|c|}{$32 \times 32$} & \multicolumn{2}{|c|}{$16 \times 16$} & \multicolumn{2}{|c|}{$8 \times 8$} \\
\hline neighborhood size & MGmix, $\mathrm{nc}=3$ & GC-MGG & MGmix, nc=3 & GC-MGG & MGmix, $\mathrm{nc}=3$ & GC-MGG & MGmix, nc=3 & GC-MGG \\
\hline $2 \times 2$ & 76.18 & 79.78 & 80.58 & 81.94 & 81.63 & 83.19 & 76.75 & 80.43 \\
\hline $2 \times 3$ & 76.39 & 81.77 & 80.65 & 83.46 & 81.10 & 83.83 & 76.62 & 79.99 \\
\hline $3 \times 2$ & 76.15 & 81.58 & 80.14 & 83.29 & 80.66 & 83.87 & 77.28 & 80.10 \\
\hline $3 \times 3$ & 77.19 & 82.66 & 80.66 & 84.31 & 81.21 & 83.72 & 76.28 & 78.29 \\
\hline $4 \times 4$ & 75.98 & 82.46 & 78.54 & 84.29 & 78.68 & 82.45 & 68.63 & 73.07 \\
\hline $5 \times 5$ & 74.69 & 81.50 & 76.95 & 83.48 & 76.63 & 80.49 & 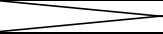 & \\
\hline
\end{tabular}

TABLE VI

COMPUTATIONAL COST (IN SECONDS) OF SIGNATURE EXTRACTION (OF AN 128X128 IMAGE) AND SIMILARITY MEASUREMENT (OF A QUERY IMAGE TO 640

\begin{tabular}{|c|c|c|c|c|c|c|c|c|c|c|}
\hline \multirow{2}{*}{ Models } & \multirow{2}{*}{ GG } & \multirow{2}{*}{ Wbl } & \multirow{2}{*}{\multicolumn{2}{|c|}{ GC-MGG }} & \multirow{2}{*}{\multicolumn{2}{|c|}{ GC-MWbl }} & \multicolumn{3}{|c|}{ MGmix } & \multirow{2}{*}{ MGG } \\
\hline & & & & & & & $\mathrm{nc}=2$ & $\mathrm{nc}=3$ & $\mathrm{nc}=4$ & \\
\hline Signature runtime & 0.06 & 0.03 & \multicolumn{2}{|c|}{0.15} & \multicolumn{2}{|c|}{0.06} & 0.44 & 0.9 & 1.7 & 1.1 \\
\hline \multirow{2}{*}{ Similarity runtime } & \multirow{2}{*}{0.15} & \multirow{2}{*}{0.17} & JD & ML & JD & ML & \multirow{2}{*}{4.9} & \multirow{2}{*}{9.1} & \multirow{2}{*}{15.5} & \multirow{2}{*}{8.7} \\
\hline & & & 2.5 & 19 & 2.5 & 12.8 & & & & \\
\hline
\end{tabular}

We propose to analyze the computational cost corresponding to the use of JD compared to the use of ML as similarity measure to retrieve images indexed using GC-MGG model. We consider a wavelet subband of a query image $I_{q}$ presented by observation matrix $\boldsymbol{X}=\left[\vec{x}_{1}, \ldots, \vec{x}_{L}\right]$ where $\vec{x}_{r}=\left(x_{1, r}, \ldots, x_{d, r}\right)^{t}, r=1, \ldots, L$ and $d=p \times q$ is the dimension of the considering neighborhood. The computational complexity of the similarity measurement between the query image and the $N_{t}$ candidate images is only function of $N_{t}, L$ and $d$. The computation of functions involved (product, summation, $\ln (\cdot), \exp (\cdot), \Gamma(\cdot), \psi(\cdot)$, $\phi^{-1}, \gamma(.,$.$) , etc.) depends on the implementation (Matlab, \mathrm{C} / \mathrm{C}++$, etc.) and on machine configuration (processor, memory, etc.). We propose to analyze the computational complexity as a function of $N_{t}, L$ and $d$, so all involved numerical functions have complexity $\mathrm{O}(1)$ (they do not depend on $N_{t}, L$ and $d$ ). We tried to perform the same optimization described in [36] consisting of precomputing of matrix inversions or determinants when image database is indexing.

Using JD between the query image $I_{q}$ and the $N_{t}$ candidate images which are indexed using GC-MGG model, requires first the estimation of hyperparameters to model the observation matrix of $I_{q}$. The estimate of marginal parameters $(\alpha, \beta)$ using $\mathrm{ML}$ estimator has a complexity of $\mathrm{O}(L)$; it does not depend neither on $N_{t}$ nor on $d$. Then, we transform the observation matrix $\boldsymbol{X}$ into Gaussian realizations $\boldsymbol{G}=\left[\vec{y}_{1}, \ldots, \vec{y}_{L}\right]$ (as described in section III-B). This Gaussianization procedure has the complexity $\mathrm{O}(d \times L)$. Finally, the estimate of $\Sigma$ using Gaussian realizations $G$ has a complexity $\mathrm{O}\left(d^{2} \times L\right)$, and $\mathrm{O}\left(d^{3}\right)$ is the complexity of calculating $\Sigma^{-1}$ using Gaussian-Jordan elimination. The whole complexity to estimate the hyperparameters of the query image is $\mathrm{O}\left(\left(d^{2}+d+1\right) \times L+d^{3}\right)$. The next step is to use the closed form of JD in equation (15) to compute similarity. This closed form does not depend on $L$, unlike ML similarity, it is independent from the observation matrix $\boldsymbol{X}$. The complexity of the JD depends only on $d$ because the covariance matrices of candidate images and their inverse are already precomputed. So, its complexity is the complexity of two matrix multiplications and two trace functions which is $\mathrm{O}\left(2\left(d^{3}+d\right)\right)$. Then, the complexity of the use of JD closed form to measure similarity between $I_{q}$ and the $N_{t}$ candidate images of the database has 
TABLE VII

AVERAGE RETRIEVAL RATES (\%) AND COMPUTATIONAL COST TO MEASURE SIMILARITIES BETWEEN EACH IMAGE IN EB 1 AND THE CANDIDATE IMAGES, USING

\begin{tabular}{|c|c|c|c|c|c|c|c|c|c|c|}
\hline & \multicolumn{2}{|c|}{ GC-MGG with JD } & \multicolumn{2}{|c|}{$\begin{array}{l}\text { GC-MGG with ML, } \\
\text { without subsampling }\end{array}$} & \multicolumn{2}{|c|}{$\begin{array}{c}\text { GC-MGG with ML, } \\
\text { subsampling factor=1/4 }\end{array}$} & \multicolumn{2}{|c|}{$\begin{array}{c}\text { GC-MGG with ML, } \\
\text { subsampling factor=1/8 }\end{array}$} & \multicolumn{2}{|c|}{$\begin{array}{c}\text { GC-MGG with ML, } \\
\text { subsampling factor=1/16 }\end{array}$} \\
\hline & ARR & Time & ARR & Time & ARR & Time & ARR & Time & $\mathrm{ARR}$ & Time \\
\hline 1 scale & 79.78 & $\sim 284 \mathrm{~s}$ & 73.82 & $\sim 11 \mathrm{~h} 14 \mathrm{~m}$ & 73.90 & $\sim 3 \mathrm{~h} 36 \mathrm{~m}$ & 73.57 & $\sim 1 \mathrm{~h} 56 \mathrm{~m}$ & 70.96 & $50 \mathrm{~m}$ \\
\hline 2 scales & 81.94 & $\sim 560 \mathrm{~s}$ & 76.15 & $\sim 14 \mathrm{~h} 38 \mathrm{~m}$ & 76.44 & $\sim 4 \mathrm{~h} 40 \mathrm{~m}$ & 76.26 & $\sim 2 \mathrm{~h} 42 \mathrm{~m}$ & 73.63 & $\sim 1 \mathrm{~h} 35 \mathrm{~m}$ \\
\hline 3 scales & 83.19 & $\sim 835 \mathrm{~s}$ & 77.27 & $\sim 15 \mathrm{~h} 40 \mathrm{~m}$ & 77.68 & $\sim 5 \mathrm{~h} 14 \mathrm{~m}$ & 77.43 & $\sim 3 \mathrm{~h} 10 \mathrm{~m}$ & 75.14 & $\sim 2 \mathrm{~h} 2 \mathrm{~m}$ \\
\hline
\end{tabular}

complexity $\mathrm{O}\left(2\left(d^{3}+d\right) \times N_{t}\right)$.

Finally, the overall complexity including estimation of the hyperparameters of the query and the use of JD closed form to measure similarity to image database is

$$
\mathrm{O}\left(2\left(d^{3}+d\right) \times N_{t}+\left(d^{2}+d+1\right) \times L+d^{3}\right)
$$

ML similarity measurement requires evaluating the log-likelihood of all vector samples $\vec{x}_{1}, \ldots, \vec{x}_{L}$ under all $N_{t}$ candidate models in the database. Considering the expression of the GC-MGG PDF in equation (11), the computational complexity of ML measurement for each candidate model consists of:

- $\mathrm{O}(d \times L)$ for the Gaussianization procedure to obtain the transformed vectors $\vec{y}_{1}, \ldots, \vec{y}_{L}$ using the marginal parameters of the candidate model.

- $\mathrm{O}\left(\left(d^{2}+d\right) \times L\right)$ for calculating $-\vec{y}_{i}^{t}\left(\Sigma^{-1}-I\right) \vec{y}_{i}, i=1, \ldots, L$.

- $\mathrm{O}(d \times L)$ for calculating $-\sum_{j=1}^{d}\left(\frac{\left|x_{i j}\right|}{\alpha}\right)^{\beta}, i=1, \ldots, L$.

The overall computational complexity of ML measurement between the query image and all $N_{t}$ candidate models in the database is then

$$
\mathrm{O}\left(\left(3 d+d^{2}\right) \times L \times N_{t}\right)
$$

We remark that ML measurement is more computationally demanding than the use of the JD closed form. For example, with a neighborhood of size $d=2 \times 2=4$, and a subband of size $L=64 \times 64=4096$, to measure a similarity of a query image to $N_{t}=1000$ candidates, we need 114688000 operations using ML, while only 222080 operations is required when using JD closed form. Even if the number of coefficients $L$ can be reduced by a factor of $1 / n$ using uniform subsampling as mentioned in [36], the ML measurement still has a considerable computational complexity $\mathrm{O}\left(\left(3 d+d^{2}\right) \times L / n \times N_{t}\right)$.

We repeated experiments on $E B 1$ using different subsampling factors $(1 / 4,1 / 8$ and 1/16) to compare the computational time of using JD or ML as similarity measure in Matlab environment. We observe that the use of JD is by far more lightweight than the use of ML. We further observe that the use of JD outperforms the use of ML similarity in term of ARR even if all data set is used without subsampling (Table VII). 


\section{CONCLUSIONS}

In this paper, a multivariate stochastic modeling approach for wavelet subbands using Gaussian copula has been presented. Extending previous works, we provide two sub-models depending on the marginal density definition: Generalized Gaussian or Weibull. The advantage of using Gaussian Copula-based models over the existing univariate cases is that they enable to incorporate correlations between wavelet coefficients while keeping a good fit to the marginal distributions. The Gaussian Copula framework exhibits flexibility for modeling a wide variety of multidimensional data. Furthermore, for such multivariate models, we have derived the closed forms of Kullback-Leibler and Jeffrey divergences in order to derive efficient similarity measures for indexing or classification applications. In the retrieval context, experimental results performed on distinct databases show that the proposed models GC-MGG and GC-MWbl provide significant improvement of performance compared to state-ofthe-art methods.

We conclude that the combined use of Gaussian Copula-based modeling and Jeffrey divergence as a similarity measure, improves retrieval performance compared to univariate modeling or the multivariate models MGmix and MGG. We can extend the proposed approach for texture segmentation and future research includes derivation of Bayesian wavelet estimator for image denoising using Gaussian Copula-based models.

\section{ACKNOWLEDGMENT}

The authors like to think Dr. Nick Kingsbury for providing the MATLAB code to the DT-CWT and Dr. Geert Verdoolaege for his code to estimate MGG distributions. The full MATLAB code for our work is available under http://sites.google.com/site/nolasmar/demo.

\section{APPENDIX}

The KLD between two Gaussian Copula-based multivariate densities $f\left(\vec{x} ; \theta_{1}\right)$ and $g\left(\vec{x} ; \theta_{2}\right)$ is given by (14).

We have

$$
f\left(\vec{x} ; \theta_{1}\right)=\frac{1}{\left|\Sigma_{1}\right|^{1 / 2}} \exp \frac{-\vec{u}^{t}\left(\Sigma_{1}^{-1}-I\right) \vec{u}}{2} \prod_{i=1}^{d} f_{i}\left(x_{i} ; \eta_{i}^{(1)}\right)
$$

where $\theta_{1}=\left\{\left(\eta_{1}^{(1)}, \ldots, \eta_{d}^{(1)}\right), \Sigma_{1}\right\}$ and $\vec{u}^{t}=\left(u_{1}, \ldots, u_{d}\right), u_{i}=\phi^{-1}\left(F_{i}\left(x_{i} ; \eta_{i}^{(1)}\right)\right), i=1, \ldots, d$.

$$
g\left(\vec{x} ; \theta_{2}\right)=\frac{1}{\left|\Sigma_{2}\right|^{1 / 2}} \exp \frac{-\vec{v}^{t}\left(\Sigma_{2}^{-1}-I\right) \vec{v}}{2} \prod_{i=1}^{d} g_{i}\left(x_{i} ; \eta_{i}^{(2)}\right)
$$

where $\theta_{2}=\left\{\left(\eta_{1}^{(2)}, \ldots, \eta_{d}^{(2)}\right), \Sigma_{2}\right\}$ and $\vec{v}^{t}=\left(v_{1}, \ldots, v_{d}\right), v_{i}=\phi^{-1}\left(G_{i}\left(x_{i} ; \eta_{i}^{(2)}\right)\right), i=1, \ldots, d$.

Hence, 


$$
\begin{aligned}
K L D\left(f\left(\vec{x} ; \theta_{1}\right) \| g\left(\vec{x} ; \theta_{2}\right)\right) & =\int \ldots \int f\left(\vec{x} ; \theta_{1}\right) \log \frac{f\left(\vec{x} ; \theta_{1}\right)}{g\left(\vec{x} ; \theta_{2}\right)} d \vec{x} \\
& =\int \ldots \int f\left(\vec{x} ; \theta_{1}\right) \log \frac{\left|\Sigma_{2}\right|^{1 / 2}}{\left|\Sigma_{1}\right|^{1 / 2}} d \vec{x}-\int \ldots \int \frac{\vec{u}^{t}\left(\Sigma_{1}^{-1}-I\right) \vec{u}}{2} f\left(\vec{x} ; \theta_{1}\right) d \vec{x}+\int \ldots \int \frac{\vec{v}^{t}\left(\Sigma_{2}^{-1}-I\right) \vec{v}}{2} f\left(\vec{x} ; \theta_{1}\right) d \vec{x} \\
& +\int \ldots \int f\left(\vec{x} ; \theta_{1}\right) \log \frac{\prod_{i=1}^{d} f_{i}\left(x_{i} ; \eta_{i}^{(1)}\right)}{\prod_{i=1}^{d} g_{i}\left(x_{i} ; \eta_{i}^{(2)}\right)} d \vec{x}
\end{aligned}
$$

On another hand we have

a)

$$
\int \ldots \int f\left(\vec{x} ; \theta_{1}\right) \log \frac{\left|\Sigma_{2}\right|^{1 / 2}}{\left|\Sigma_{1}\right|^{1 / 2}} d \vec{x}=\log \frac{\left|\Sigma_{2}\right|^{1 / 2}}{\left|\Sigma_{1}\right|^{1 / 2}} \times \int \ldots \int f\left(\vec{x} ; \theta_{1}\right) d \vec{x}=\log \frac{\left|\Sigma_{2}\right|^{1 / 2}}{\left|\Sigma_{1}\right|^{1 / 2}}
$$

b)

$$
\begin{gathered}
\int \ldots \int \frac{\vec{u}^{t}\left(\Sigma_{1}^{-1}-I\right) \vec{u}}{2} f\left(\vec{x} ; \theta_{1}\right) d \vec{x}=\operatorname{trace}\left(\int \ldots \int \frac{\vec{u}^{t}\left(\Sigma_{1}^{-1}-I\right) \vec{u}}{2} f\left(\vec{x} ; \theta_{1}\right) d \vec{x}\right) \\
=0.5 \int \ldots \int \operatorname{trace}\left(\vec{u}^{t}\left(\Sigma_{1}^{-1}-I\right) \vec{u} f\left(\vec{x} ; \theta_{1}\right)\right) d \vec{x} \\
=0.5 \operatorname{trace}\left(\int \ldots \int f\left(\vec{x} ; \theta_{1}\right)\left(\Sigma_{1}^{-1}-I\right) \vec{u} \vec{u}^{t} d \vec{x}\right) \\
=0.5 \operatorname{trace}\left(\left(\Sigma_{1}^{-1}-I\right) \int \ldots \int f\left(\vec{x} ; \theta_{1}\right) \vec{u} \vec{u}^{t} d \vec{x}\right)
\end{gathered}
$$

While $\int \ldots \int f\left(\vec{x} ; \theta_{1}\right) \vec{u} \vec{u}^{t} d \vec{x}=\int \ldots \int N\left(\vec{u} ; 0, \Sigma_{1}\right) \vec{u} \vec{u}^{t} d \vec{u}=\Sigma_{1}$, where $N\left(\vec{u} ; 0, \Sigma_{1}\right)$ denotes a multivariate Gaussian distribution, (20) becomes

$$
\begin{aligned}
\int \ldots \int \frac{\vec{u}^{t}\left(\Sigma_{1}^{-1}-I\right) \vec{u}}{2} f\left(\vec{x} ; \theta_{1}\right) d \vec{x} & =0.5 \operatorname{trace}\left(\left(\Sigma_{1}^{-1}-I\right) \Sigma_{1}\right) \\
& =0
\end{aligned}
$$

c) In the same manner of b) we have

$$
\begin{aligned}
& \int \ldots \int \frac{\vec{v}^{t}\left(\Sigma_{2}^{-1}-I\right) \vec{v}}{2} f\left(\vec{x} ; \theta_{1}\right) d \vec{x} \\
& =0.5 \operatorname{trace}\left(\left(\Sigma_{2}^{-1}-I\right) \int \ldots \int f\left(\vec{x} ; \theta_{1}\right) \vec{v} \vec{v}^{t} d \vec{x}\right) \\
& =0.5 \operatorname{trace}\left(\left(\Sigma_{2}^{-1}-I\right) \Sigma_{1}\right)=0.5\left(\operatorname{trace}\left(\Sigma_{2}{ }^{-1} \Sigma_{1}\right)-d\right)
\end{aligned}
$$

d)

$$
\int \ldots \int f\left(\vec{x} ; \theta_{1}\right) \log \frac{\prod_{i=1}^{d} f_{i}\left(x_{i} ; \eta_{i}^{(1)}\right)}{\prod_{i=1}^{d} g_{i}\left(x_{i} ; \eta_{i}^{(2)}\right)} d \vec{x}=\sum_{i=1}^{d} \int \ldots \int f\left(\vec{x} ; \theta_{1}\right) \log \frac{f_{i}\left(x_{i} ; \eta_{i}^{(1)}\right)}{g_{i}\left(x_{i} ; \eta_{i}^{(2)}\right)} d \vec{x}
$$

Since

$$
\int \ldots \int f\left(\vec{x} ; \theta_{1}\right) \log \frac{f_{i}\left(x_{i} ; \eta_{i}^{(1)}\right)}{g_{i}\left(x_{i} ; \eta_{i}^{(2)}\right)} d \vec{x}=\int f_{i}\left(x_{i} ; \eta_{i}^{(1)}\right) \log \frac{f_{i}\left(x_{i} ; \eta_{i}^{(1)}\right)}{g_{i}\left(x_{i} ; \eta_{i}^{(2)}\right)} d \vec{x}_{i}
$$


Then (23) becomes

$$
\int \ldots \int f\left(\vec{x} ; \theta_{1}\right) \log \frac{\prod_{i=1}^{d} f_{i}\left(x_{i} ; \eta_{i}^{(1)}\right)}{\prod_{i=1}^{d} g_{i}\left(x_{i} ; \eta_{i}^{(2)}\right)} d \vec{x}=\sum_{i=1}^{d} K L D\left(f_{i}\left(x_{i} ; \eta_{i}^{(1)}\right) \| g_{i}\left(x_{i} ; \eta_{i}^{(2)}\right)\right)
$$

Finally, we sum up (19), (21), (22) and (24) to obtain

$$
K L D\left(f\left(\vec{x} ; \theta_{1}\right) \| g\left(\vec{x} ; \theta_{2}\right)\right)=\sum_{i=1}^{d} K L D\left(f_{i}\left(x_{i} ; \eta_{i}^{(1)}\right) \| g_{i}\left(x_{i} ; \eta_{i}^{(2)}\right)\right)+0.5\left(\operatorname{tr}\left(\Sigma_{2}^{-1} \Sigma_{1}\right)+\log \frac{\left|\Sigma_{2}\right|}{\left|\Sigma_{1}\right|}-d\right)
$$

\section{REFERENCES}

[1] N. Vasconcelos and A. Lippman, "A unifying view of image similarity," in Proceedings of the 15th International Conference on Pattern Recognition (ICPR'00), pp. 38-41, 2000.

[2] M. Do and M. Vetterli, "Wavelet-based texture retrieval using generalized Gaussian density and Kullback-Leibler distance," IEEE Transactions on Image Processing, vol. 11, no. 2, pp. 146-158, Feb. 2002.

[3] R. Haralick, "Statistical and structural approaches to texture," Proceedings of the IEEE, vol. 67, n. 5, pp. 786-804, May. 1979.

[4] I. M. Elfadel and R. W. Picard, "Gibbs random fields, cooccurrences, and texture modeling," IEEE Transactions on Pattern Analysis and Machine Intelligence, vol. 16, $\mathrm{n}^{\circ} .1$, pp. 24-37, Jan. 1994.

[5] G. Lohmann, “Analysis and synthesis of textures: A co-occurrence-based approach,” Computers \& Graphics, vol. 19, n ${ }^{\circ}$ 1, pp. $29-36$, Jan. 1995.

[6] A. Srivastava, X. Liu, and U. Grenander, "Universal analytical forms for modeling image probabilities," IEEE Transactions on Pattern Analysis and Machine Intelligence, vol. 24, no. 9, pp. 1200-1214, Sep. 2002.

[7] J. R. Mathiassen, A. Skavhaug, and K. Bø, "Texture Similarity Measure Using Kullback-Leibler Divergence between Gamma Distributions," in Proceedings of the 7th European Conference on Computer Vision (ECCV'O2), pp. 133-147, 2002.

[8] R. Kwitt and A. Uhl, "Image similarity measurement by Kullback-Leibler divergences between complex wavelet subband statistics for texture retrieval," in Proceedings of the 15th IEEE International Conference on Image Processing (ICIP'08), pp. 933-936, 2008.

[9] R. Kwitt and A. Uhl, “Lightweight Probabilistic Texture Retrieval,” IEEE Transactions on Image Processing, vol. 19, no. 1, pp. 241-253, Jan. 2010.

[10] A. K. Jain and F. Farrokhnia, “Unsupervised texture segmentation using Gabor filters,” Pattern Recognition, vol. 24, no. 12, pp. 1167-1186, Dec. 1991.

[11] A. Laine and J. Fan, “Texture classification by wavelet packet signatures,” IEEE Transactions on Pattern Analysis and Machine Intelligence, vol. 15, no. 11, pp. 1186-1191, Nov. 1993.

[12] M. Unser, "Texture classification and segmentation using wavelet frames," IEEE Transactions on Image Processing, vol. 4, no. 11, pp. 1549-1560, Nov. 1995.

[13] T. Randen and J. Husoy, "Filtering for texture classification: a comparative study," IEEE Transactions on Pattern Analysis and Machine Intelligence, vol. 21, no. 4, pp. 291-310, Apr. 1999.

[14] H. Tamura, S. Mori, and T. Yamawaki, "Textural Features Corresponding to Visual Perception," IEEE Transactions on Systems, Man, and Cybernetics, vol. 8 , no. 6 , pp. $460-473,1978$.

[15] J. Daugman, "Uncertainty relation for resolution in space, spatial frequency, and orientation optimized by two-dimensional visual cortical filters," Journal of the Optical Society of America, vol. 2, no. 7, pp. 1160-1169, Jul. 1985.

[16] K. Fujii, S. Sugi, and Y. Ando, "Textural properties corresponding to visual perception based on the correlation mechanism in the visual system," Psychological Research, vol. 67, pp. 197-208, 2003. 
[17] M. S. Landy and N. Graham, "Visual perception of texture," in The Visual Neurosciences, L. M. Chalupa and J. S. Werner, Eds. Cambridge, MA: MIT Press, 2004, pp. 1106-1118.

[18] S. Mallat, "A theory for multiresolution signal decomposition: the wavelet representation," IEEE Transactions on Pattern Analysis and Machine Intelligence, vol. 11, no. 7, pp. 674-693, Jul. 1989.

[19] G. Van de Wouwer, P. Scheunders, and D. Van Dyck, "Statistical texture characterization from discrete wavelet representations," IEEE Transactions on Image Processing, vol. 8, no. 4, pp. 592-598, Apr. 1999.

[20] G. Tzagkarakis, B. Beferull-Lozano, and P. Tsakalides, "Rotation invariant texture retrieval with Gaussianized Steerable Pyramids," IEEE Transactions on Image Processing, vol. 15, no. 9, pp. 2702-2718, Sep. 2006.

[21] D. Cho et T. D. Bui, "Multivariate statistical modeling for image denoising using wavelet transforms," Signal Processing: Image Communication, vol. 20, $\mathrm{n}^{\circ} .1$, pp. 77-89, Jan. 2005.

[22] S. Tan and L. Jiao, "Multivariate Statistical Models for Image Denoising in the Wavelet Domain," International Journal of Computer Vision, vol. 75, no. 2, pp. 209-230, Nov. 2007.

[23] M. J. Wainwright and E. P. Simoncelli, "Scale Mixtures of Gaussians and the Statistics of Natural Images," Advances in Neural Information Processing Systems, vol. 12, pp. 855-861, 2000.

[24] J. Portilla, V. Strela, M. Wainwright, and E. Simoncelli, "Image denoising using scale mixtures of gaussians in the wavelet domain," IEEE Transactions on Image Processing, vol. 12, no. 11, pp. 1338-1351, Nov. 2003.

[25] L. Boubchir, A. Nait-Ali, and E. Petit, "Multivariate statistical modeling of images in sparse multiscale transforms domain," in Proceedings of the 17th IEEE International Conference on Image Processing (ICIP'10), Hong Kong, Hong Kong, pp. 1877-1880, 2010.

[26] N. Vasconcelos and A. Lippman, “A probabilistic architecture for content-based image retrieval,” in Proceedings of IEEE Conference on Computer Vision and Pattern Recognition (CVPR'00), pp. 216-221, 2000.

[27] Goldberger, Gordon, and Greenspan, "An efficient image similarity measure based on approximations of KL-divergence between two gaussian mixtures," in Proceedings of the 9th IEEE International Conference on Computer Vision (ICCV'03), pp. 487-493 vol.1, 2003.

[28] N. Vasconcelos, “On the Efficient Evaluation of Probabilistic Similarity Functions for Image Retrieval," IEEE Transactions on Information Theory, vol. 50, no. 7, pp. 1482-1496, Jul. 2004.

[29] H. Permuter, J. Francos, and I. Jermyn, "A study of Gaussian mixture models of color and texture features for image classification and segmentation," Pattern Recognition, vol. 39, no. 4, pp. 695-706, Apr. 2006.

[30] S. C. Kim and T. J. Kang, “Texture classification and segmentation using wavelet packet frame and Gaussian mixture model,” Pattern Recognition, vol. 40, n. 4, pp. 1207-1221, Avr. 2007

[31] J. Hershey and P. Olsen, "Approximating the Kullback Leibler Divergence between Gaussian Mixture Models," in Proceedings of IEEE International Conference on Acoustics, Speech and Signal Processing (ICASSP'07), pp. 317-320.

[32] G. Verdoolaege and P. Scheunders, "Geodesics on the Manifold of Multivariate Generalized Gaussian Distributions with an Application to Multicomponent Texture Discrimination,” International Journal of Computer Vision, vol. 95, no. 3, pp. 265-286, May 2011.

[33] S. Sakji-Nsibi and A. Benazza-Benyahia, "Copula-based statistical models for multicomponent image retrieval in the wavelet transform domain," in Proceedings of the 16th IEEE International Conference on Image Processing (ICIP'09), pp. 253-256, 2009.

[34] R. Kwitt and A. Uhl, “A joint model of complex wavelet coefficients for texture retrieval,” in Proceedings of the 16th IEEE International Conference on Image Processing (ICIP'09), pp. 1877-1880, 2009.

[35] S. Sakji-Nsibi and A. Benazza-Benyahia, "Fast scalable retrieval of multispectral images with Kullback-Leibler divergence," in Proceedings of the 17th IEEE International Conference on Image Processing (ICIP'10), pp. 2333-2336, 2010. 
[36] R. Kwitt, P. Meerwald, and A. Uhl, "Efficient Texture Image Retrieval Using Copulas in a Bayesian Framework," IEEE Transactions on Image Processing, vol. 20, no. 7, pp. 2063-2077, Jul. 2011.

[37] Y. Stitou, N. Lasmar, and Y. Berthoumieu, "Copulas based multivariate gamma modeling for texture classification," in Proceedings of IEEE International Conference on Acoustics, Speech and Signal Processing (ICASSP'09), pp. 1045-1048, 2009.

[38] H. Liu, D. Song, S. Rüger, R. Hu, and V. Uren, “Comparing Dissimilarity Measures for Content-Based Image Retrieval," in Information Retrieval Technology, vol. 4993, H. Li, T. Liu, W. Ma, T. Sakai, K. Wong, and G. Zhou, Eds. Springer Berlin / Heidelberg, 2008, pp. 44-50.

[39] D. D. Po and M. N. Do, "Directional multiscale modeling of images using the contourlet transform," IEEE Transactions on Image Processing, vol. 15, no. 6, pp. 1610-1620, Jun. 2006.

[40] N. I. Fisher and P. Switzer, “Graphical assessment of dependence: Is a picture worth 100 tests?,” The American statistician, vol. 55, no. 3, pp. $233-239$.

[41] T. M. Cover and J. A. Thomas, Elements of Information Theory. New York: Wiley-Interscience, 1991.

[42] U. Cherubini, E. Luciano, and W. Vecchiato, Copula Methods in Finance, New York: Wiley, 2004.

[43] G. Escarela and J. F. Carriere, "Fitting competing risks with an assumed copula," Statistical Methods in Medical Research, vol. 12, no. 4, pp. 333 -349, 2003.

[44] C. Genest and A. Favre, "Everything you always wanted to know about copula modeling but were afraid to ask," Journal of Hydrologic Engineering, vol. 12, no. 4, pp. 347--368, Jul. 2007.

[45] R. B. Nelsen, An Introduction to Copulas, 2nd ed. New York: Springer-Verlag, 2006.

[46] A. Sklar, "Random variables, joint distribution functions, and copulas," Kybernetika, vol. 9, no. 6, pp. 449-460, 1973.

[47] I. Zezula, “On multivariate Gaussian copulas,” Journal of Statistical Planning and Inference, vol. 139, nº 11, pp. 3942-3946, Nov. 2009.

[48] H. Joe and J. J. Xu, "the estimation method of inference functions for margins for multivariate models," Technical Report no. 166, Department of Statistics, University of British Columbia, 1996.

[49] M. Abramowitz and I. A. Stegun, Handbook of Mathematical Functions: with Formulas, Graphs, and Mathematical Tables. New York: Dover Publications, 1965.

[50] N. L. Johnson, S. Kotz, and N. Balakrishnan, Continuous Univariate Distributions, Vol. 1, 2nd ed. Wiley-Interscience, 1994.

[51] MIT Vision and Modeling Group. Vision Texture. [Online]. Available: http://vismod.www.media.mit.edu

[52] Brodatz Texture Images [Online], Available: http://www.ux.uis.no/ tranden/brodatz.html

[53] Amsterdam Library of Textures. [Online]. Available: http://staff.science.uva.nl/ aloi/public alot

[54] Salzburg Textures [Online]. Available: http://wavelab.at/sources/STex

[55] I. Daubechies, Ten lectures on wavelets. Philadelphia, PA, USA: Society for Industrial and Applied Mathematics, 1992.

[56] N. Kingsbury, "Complex Wavelets for Shift Invariant Analysis and Filtering of Signals," Applied and Computational Harmonic Analysis, vol. 10, no. 3, pp. 234-253, May. 2001.

[57] H. Müller, W. Müller, D. M. Squire, S. Marchand-Maillet, and T. Pun, "Performance evaluation in content-based image retrieval: overview and proposals," Pattern Recognition Letters, vol. 22, no. 5, pp. 593-601, Apr. 2001.

[58] A. Singhal, "Modern Information Retrieval: A Brief Overview," Bulletin of the IEEE Computer Society Technical Committee on Data Engineering, vol. 24, no. 4, pp. 35-42, 2001.

[59] L. Bombrun, Y. Berthoumieu, N.-E. Lasmar, and G. Verdoolaege, "Multivariate texture retrieval using the geodesic distance between elliptically distributed random variables," in Image Processing (ICIP), 2011 18th IEEE International Conference on, 2011, pp. 3637 -3640.

[60] W. D. Penny, "Kl-divergences of normal, gamma, dirichlet and wishart densities," Technical Report, Wellcome Department of Cognitive Neurology, 2001. 


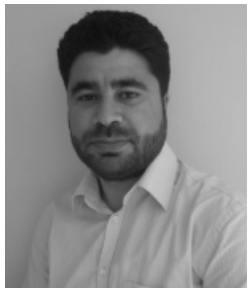

Nour-Eddine. Lasmar (S’09) received the M.Sc. degree (with first class honors) in computer sciences \& telecommunications from the University of Sciences, Rabat, Morocco, in 2007, and the Ph.D degree in Signal \& image processing from the University of Bordeaux I, France, in 2012.

He is currently a research engineer with ENSTA Bretagne, Brest, France. His research interests include stochastic modeling, texture image analysis and machine learning.

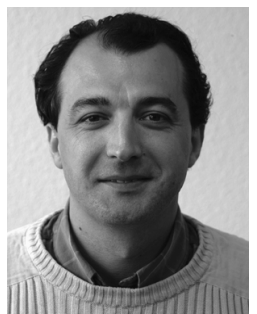

Yannick Berthoumieu (M’09) received the Ph.D degree in signal processing from the University of Bordeaux I, France, in 1996.

In 1998, he joined the ENSEIRB (Ecole Nationale Supérieure d'Electronique, Informatique et Radiocommunications de Bordeaux) as an Assistant Professor. He is currently a Professor with the Department of Telecommunications, Institut Polytechnique de Bordeaux, and University of Bordeaux.

His major research interest includes multidimensional signal analysis, stochastic modeling, texture analysis, and image and video processing. Since 2003, he is with the joint laboratory LASIS between the LAPS department and the Total company within the joint research laboratory IMS UMR 5218 